This item was submitted to Loughborough's Research Repository by the author.

Items in Figshare are protected by copyright, with all rights reserved, unless otherwise indicated.

\title{
On the integrable elliptic cylindrical Kadomtsev-Petviashvili equation
}

PLEASE CITE THE PUBLISHED VERSION

http://dx.doi.org/10.1063/1.4792268

\section{PUBLISHER}

(C) American Institute of Physics

VERSION

VoR (Version of Record)

LICENCE

CC BY-NC-ND 4.0

REPOSITORY RECORD

Khusnutdinova, Karima R., C. Klein, Vladimir S. Matveev, and A.O. Smirnov. 2019. "On the Integrable Elliptic Cylindrical Kadomtsev-petviashvili Equation”. figshare. https://hdl.handle.net/2134/15723. 


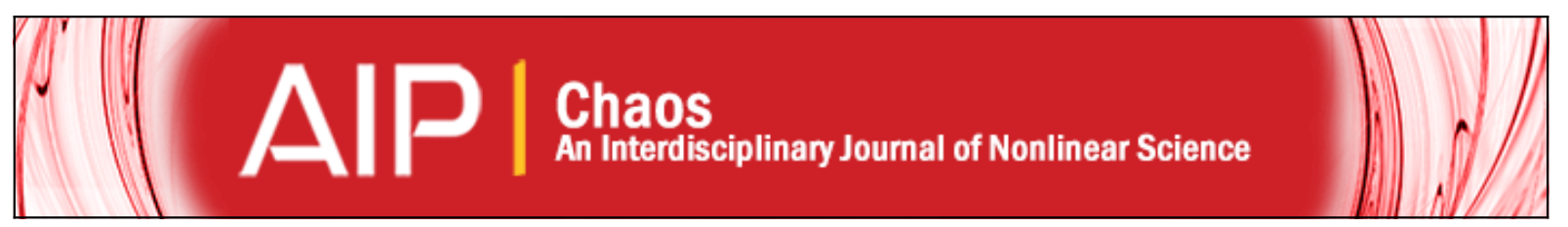

\section{On the integrable elliptic cylindrical Kadomtsev-Petviashvili equation}

K. R. Khusnutdinova, C. Klein, V. B. Matveev, and A. O. Smirnov

Citation: Chaos: An Interdisciplinary Journal of Nonlinear Science 23, 013126 (2013); doi: 10.1063/1.4792268

View online: http://dx.doi.org/10.1063/1.4792268

View Table of Contents: http://scitation.aip.org/content/aip/journal/chaos/23/1 ?ver=pdfcov

Published by the AIP Publishing

\section{Articles you may be interested in}

Integrability properties of the dispersionless Kadomtsev-Petviashvili hierarchy

J. Math. Phys. 55, 083504 (2014); 10.1063/1.4890480

Algebro-geometric solutions of (2+1) -dimensional coupled modified Kadomtsev-Petviashvili equations

J. Math. Phys. 41, 337 (2000); 10.1063/1.533134

Higher dimensional Painlevé integrable models from the Kadomtsev-Petviashvili equation

J. Math. Phys. 39, 5364 (1998); 10.1063/1.532576

On solutions of constrained Kadomtsev-Petviashvili equations: Grammians

J. Math. Phys. 38, 5190 (1997); 10.1063/1.531937

Algebra of pseudodifferential operators and symmetries of equations in the Kadomtsev-Petviashvili hierarchy

J. Math. Phys. 38, 4644 (1997); 10.1063/1.532112

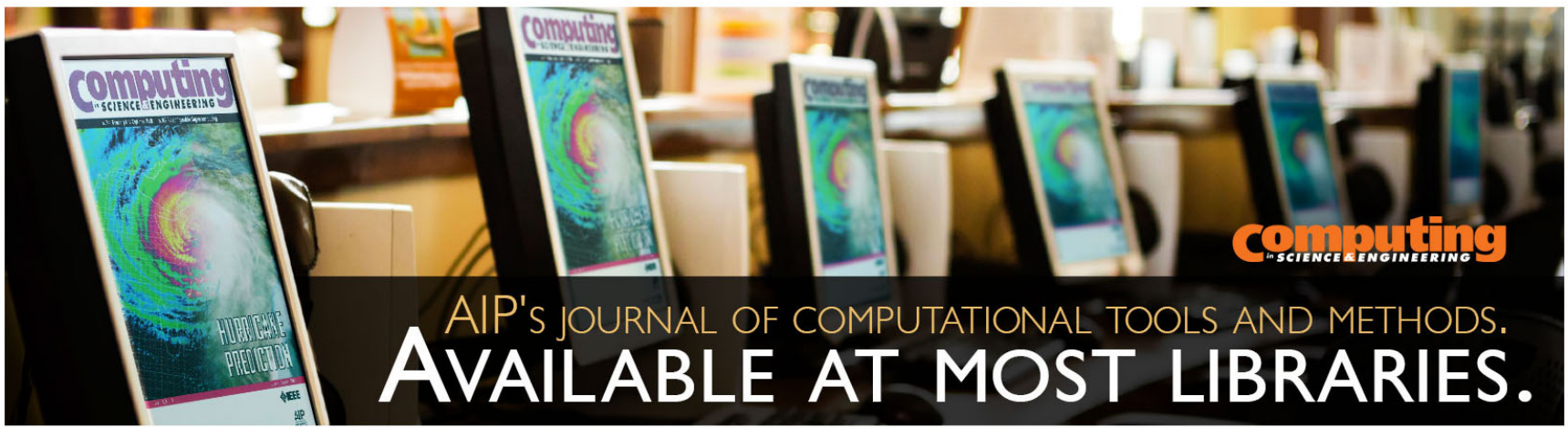




\title{
On the integrable elliptic cylindrical Kadomtsev-Petviashvili equation
}

\author{
K. R. Khusnutdinova, ${ }^{1, a)}$ C. Klein, ${ }^{2}$ V. B. Matveev, ${ }^{2}$ and A. O. Smirnov ${ }^{3}$ \\ ${ }^{1}$ Department of Mathematical Sciences, Loughborough University, Loughborough LE11 3TU, United Kingdom \\ ${ }^{2}$ Institut de Mathématiques de Bourgogne, Université de Bourgogne, BP 47870, F-21078 Dijon Cedex, France \\ ${ }^{3}$ St. Petersburg University of Aerospace Instrumentation, St. Petersburg 190000, Russia
}

(Received 9 November 2012; accepted 31 January 2013; published online 21 February 2013)

There exist two versions of the Kadomtsev-Petviashvili (KP) equation, related to the Cartesian and cylindrical geometries of the waves. In this paper, we derive and study a new version, related to the elliptic cylindrical geometry. The derivation is given in the context of surface waves, but the derived equation is a universal integrable model applicable to generic weakly nonlinear weakly dispersive waves. We also show that there exist nontrivial transformations between all three versions of the KP equation associated with the physical problem formulation, and use them to obtain new classes of approximate solutions for water waves. (C) 2013 American Institute of Physics. [http://dx.doi.org/10.1063/1.4792268]

The "elliptic cylindrical Kadomtsev-Petviashvili (ecKP) equation,"

$$
\begin{aligned}
& \left(H_{\tau}+6 H H_{\zeta}+H_{\zeta \zeta \zeta}+\frac{\tau}{2\left(\tau^{2}-a^{2}\right)} H-\frac{a^{2} \nu^{2}}{12 \sigma^{2}\left(\tau^{2}-a^{2}\right)} H_{\zeta}\right)_{\zeta} \\
& \quad+\frac{3 \sigma^{2}}{\tau^{2}-a^{2}} H_{\nu \nu}=0,
\end{aligned}
$$

where $a$ is a parameter and $\sigma^{2}= \pm 1$, is derived for surface gravity waves with nearly elliptic front, generalising the cylindrical KP equation for nearly concentric waves and describing the intermediate asymptotics. We find transformations between the derived ecKP equation and two existing versions of the KP equation for water wave problems, for nearly plane and nearly concentric waves, as well as the Lax pair for the ecKP equation. The transformations are used to construct important classes of exact solutions of the derived ecKP equation and corresponding new asymptotic solutions for the Euler equations from the known solutions of the $\mathrm{KP}$ equation. The ecKP equation is a universal integrable model applicable to generic weakly nonlinear weakly dispersive waves with nearly elliptic wave fronts.

\section{INTRODUCTION}

There exist two classical versions of the KP equation ${ }^{1}$ associated with the surface wave problems for an incompressible fluid, described by the full set of Euler equations with free surface and rigid horizontal bottom boundary conditions (see, Refs. 2-5 and references therein)

\footnotetext{
a) Author to whom correspondence should be addressed. E-mail: K.Khusnutdinova@lboro.ac.uk. Tel.: +44 (0)1509 228202. Fax: +44 (0)1509223969.
}

$$
\begin{aligned}
& \rho\left(u_{t}+u u_{x}+v u_{y}+w u_{z}\right)=-p_{x}, \\
& \rho\left(v_{t}+u v_{x}+v v_{y}+w v_{z}\right)=-p_{y}, \\
& \rho\left(w_{t}+u w_{x}+v w_{y}+w w_{z}\right)=-p_{z}-\rho g, \\
& u_{x}+v_{y}+w_{z}=0 \\
& \left.p\right|_{z=h(x, y, t)}=p_{a} \\
& \quad-\Gamma \frac{\left(1+h_{y}^{2}\right) h_{x x}+\left(1+h_{x}^{2}\right) h_{y y}-2 h_{x} h_{y} h_{x y}}{\left(1+h_{x}^{2}+h_{y}^{2}\right)^{3 / 2}}, \\
& \left.w\right|_{z=h(x, y, t)}=h_{t}+u h_{x}+v h_{y}, \\
& \left.w\right|_{z=0}=0 .
\end{aligned}
$$

Here, $(u, v, w)$ are the three components of the velocity vector in Cartesian coordinates $(x, y, z), t$ is the time, $p$ the pressure $\left(p_{a}\right.$ is the constant atmospheric pressure at the surface, and $\Gamma$ is the coefficient of the surface tension), $\rho$ is constant density, $g$ is the gravitational acceleration, $z=0$ is the bottom, and $z=h(x, y, t)$ is the free surface. The original KP equation, ${ }^{1}$

$$
\left(U_{\tau}+6 U U_{\xi}+U_{\xi \xi \xi}\right)_{\xi}+3 \sigma^{2} U_{Y Y}=0,
$$

and the cylindrical KP (cKP) equation, ${ }^{3}$

$$
\left(W_{\tau}+6 W W_{\chi}+W_{\chi \chi \chi}+\frac{W}{2 \tau}\right)_{\chi}+\frac{3 \sigma^{2}}{\tau^{2}} W_{V V}=0,
$$

are derived for the leading order term of the asymptotic expansion of the free surface elevation in the appropriate sets of fast and slow variables, and describe the weakly nonlinear evolution of long nearly plane and nearly concentric waves, respectively. For surface gravity waves with no or weak surface tension, one has $\sigma^{2}=1$ (KP-II), while the case $\sigma^{2}=-1$ (KP-I) can be obtained when surface tension effects are large (see, for example, Ref. 6 and references therein). Useful transformations mapping solutions of the cKP and KP equations were independently found in Refs. 3, 5, and 7. They have been used to construct some special solutions of the cKP equation in Refs. 8 and 9. Indeed, the map 


$$
W(\tau, \chi, V) \rightarrow U(\tau, \xi, Y):=W\left(\tau, \xi+\frac{Y^{2}}{12 \sigma^{2} \tau}, \frac{Y}{\tau}\right)
$$

transforms any solution of the cKP Eq. (3) into a solution of the KP Eq. (2). Conversely, the map

$$
U(\tau, \xi, Y) \rightarrow W(\tau, \chi, V):=U\left(\tau, \chi-\frac{\tau V^{2}}{12 \sigma^{2}}, \tau V\right)
$$

transforms any solution of the KP Eq. (2) into a solution of the cKP Eq. (3). In Ref. 10, it was pointed out that the transformations map rather general classes of evolution equations, containing the KP and cKP equations (as well as mapping their one-dimensional counterparts generalising the $\mathrm{KdV}$ and $\mathrm{cKdV}$ equations into some classes of solutions of the twodimensional equations).

Another interesting transformation linking the cKP and KP equations was found in Ref. 11 (see also Ref. 8). However, this transformation maps bounded solutions of the KP equation into unbounded solutions of the cKP equation. We do not consider transformations of this type in our paper.

In this paper, we derive a generalisation of the cKP Eq. (3) for surface gravity waves, which can be written in the form

$$
\begin{aligned}
& \left(H_{\tau}+6 H H_{\zeta}+H_{\zeta \zeta \zeta}+\frac{\tau}{2\left(\tau^{2}-a^{2}\right)} H-\frac{\nu^{2} a^{2}}{12 \sigma^{2}\left(\tau^{2}-a^{2}\right)} H_{\zeta}\right)_{\zeta} \\
& \quad+\frac{3 \sigma^{2}}{\tau^{2}-a^{2}} H_{\nu \nu}=0,
\end{aligned}
$$

where $\sigma^{2}= \pm 1$, describing waves with nearly elliptic front. This ecKP equation is derived from the full set of Euler equations for an incompressible fluid and free surface and rigid bottom boundary conditions (1), written in the elliptic cylindrical coordinate system. The linear long-wave equation, written in these coordinates, does not allow for exact solutions describing waves with elliptic front. However, there exists an asymptotic reduction to the necessary equation, and we show that this allows one to derive a generalisation of the cKP equation.

We chose to derive the ecKP equation from the Euler equations rather than using the velocity potential formulation, since this opens the way to the study of internal and surface waves on a current for a fluid with arbitrary stratification, as well as accounting for the effects of a variable background and Earth's rotation (see, Refs. 13 and 14 and references therein, for studies in the Cartesian geometry), which constitute rotational flows.

We find transformations between KP, cKP, and ecKP equations, generalising the transformations between $\mathrm{KP}$ and cKP equations in Refs. 3, 5, and 7, and use them to construct some important special classes of solutions of the derived version of the Kadomtsev-Petviashvili equation for both cases (i.e., for ecKP-I and ecKP-II). Indeed, the map

$$
U(\tau, \xi, Y) \rightarrow H(\tau, \zeta, \nu):=U\left(\tau, \zeta-\frac{\tau \nu^{2}}{12 \sigma^{2}}, \sqrt{\tau^{2}-a^{2}} \nu\right)
$$

transforms any solution of the KP equation into a solution of the ecKP equation. Conversely, the map

$$
\begin{aligned}
& H(\tau, \zeta, \nu) \rightarrow \\
& U(\tau, \xi, Y):=H\left(\tau, \xi+\frac{1}{12 \sigma^{2}} \frac{\tau Y^{2}}{\tau^{2}-a^{2}}, \frac{Y}{\sqrt{\tau^{2}-a^{2}}}\right)
\end{aligned}
$$

transforms any solution of the ecKP equation into a solution of the KP equation.

The ecKP Eq. (4) derived in our paper is an integrable model, which can be obtained as a compatibility condition of the following linear problem (Lax pair)

$$
\begin{aligned}
& \sigma \psi_{\nu}=\sqrt{\tau^{2}-a^{2}} \psi_{\zeta \zeta}+\left(\sqrt{\tau^{2}-a^{2}} H(\tau, \zeta, \nu)\right. \\
& \left.-\frac{\tau \zeta}{12 \sqrt{\tau^{2}-a^{2}}}+\frac{a^{2} \nu^{2}}{144 \sigma^{2} \sqrt{\tau^{2}-a^{2}}}\right) \psi, \\
& \psi_{\tau}=-4 \psi_{\zeta \zeta \zeta}-\left(6 H(\tau, \zeta, \nu)-\frac{a^{2} \nu^{2}}{12 \sigma^{2}\left(\tau^{2}-a^{2}\right)}\right) \psi_{\zeta} \\
& -\left(3 H_{\zeta}(\tau, \zeta, \nu)+\frac{3 \sigma \widetilde{H}(\tau, \zeta, \nu)}{\sqrt{\tau^{2}-a^{2}}}-\frac{a^{2} \zeta \nu}{12 \sigma\left(\tau^{2}-a^{2}\right)^{3 / 2}}\right) \psi .
\end{aligned}
$$

Indeed, the compatibility conditions have the form

$$
\begin{aligned}
& \widetilde{H}_{\zeta}=H_{\nu}, \\
& H_{\tau}+6 H H_{\zeta}+H_{\zeta \zeta \zeta}+\frac{\tau}{2\left(\tau^{2}-a^{2}\right)} H \\
& -\frac{a^{2} \nu^{2}}{12 \sigma^{2}\left(\tau^{2}-a^{2}\right)} H_{\zeta}+\frac{3 \sigma^{2}}{\tau^{2}-a^{2}} \widetilde{H}_{\nu}=0 .
\end{aligned}
$$

When $a=0$, we recover the Lax pair of the cKP equation ${ }^{12}$ (see also Ref. 9).

A solution $\psi_{e c K P}(\tau, \zeta, \nu)$ of the linear system (5) and (6) is expressed via the solution $\psi_{K P}(\tau, \xi, Y)$ of the linear systems of the KP equation $\left(\widetilde{U}_{\xi}=U_{Y}\right)$

$$
\begin{aligned}
\sigma \psi_{Y}= & \psi_{\xi \xi}+U(\tau, \xi, Y) \psi, \\
\psi_{\tau}= & -4 \psi_{\xi \xi \xi}-6 U(\tau, \xi, Y) \psi_{\xi} \\
& -\left(3 U_{\xi}(\tau, \xi, Y)+3 \sigma \widetilde{U}(\tau, \xi, Y)\right) \psi
\end{aligned}
$$

as follows:

$$
\begin{aligned}
\psi_{e c K P}(\tau, \zeta, \nu)= & \psi_{K P}\left(\tau, \zeta-\frac{\tau \nu^{2}}{12 \sigma^{2}}, \sqrt{\tau^{2}-a^{2}} \nu\right) \\
& \times \exp \left\{-\frac{\zeta \tau \nu}{12 \sigma \sqrt{\tau^{2}-a^{2}}}+\frac{\nu^{3}\left(\tau^{2}+a^{2}\right)}{432 \sigma^{3} \sqrt{\tau^{2}-a^{2}}}\right\} .
\end{aligned}
$$

Let us also note that the functions $\widetilde{H}$ and $\widetilde{U}$ are related by

$$
\widetilde{H}(\tau, \zeta, \nu)=\sqrt{\tau^{2}-a^{2}} \widetilde{U}(\tau, \xi, Y)-\frac{\tau \nu}{6 \sigma^{2}} U(\tau, \xi, Y) .
$$

The Jacobian of the transformation

$$
\xi=\zeta-\tau \nu^{2} /\left(12 \sigma^{2}\right), \quad Y=\sqrt{\tau^{2}-a^{2}} \nu,
$$

used to construct some special solutions of the ecKP in the following sections, is equal to $\sqrt{\tau^{2}-a^{2}}$. It is positive and bounded for all $\tau>a$. (This condition is automatically satisfied for our derivation.)

The ecKP equation is an integrable equation containing an arbitrary parameter $a$, and it reduces to the cKP equation 
both when this parameter tends to zero, and when $\tau \gg a$. The latter is the mathematical manifestation of the intuitively clear physical phenomenon: wave fronts will become nearly concentric far away from the wave sources or boundaries, while they will "remember" their geometrical shape during the intermediate evolution.

The KP and cKP equations belong to the family of universal integrable models of modern nonlinear physics (see, for example, Refs. 4 and 15). As well as for surface waves, these equations have been derived in many physical settings (see, for example, Ref. 6), including internal waves in a stratified fluid ${ }^{16,17}$ and, most recently, matter waves in Bose-Einstein condensates (BEC) (see, Refs. 18 and 19 and references therein) and cosmic dustion-acoustic waves. ${ }^{20}$ Thus, the new version of the equation derived in this paper could find many useful applications to the description of the wave motion in problems where sources, boundaries, and obstacles have elliptic or nearly elliptic geometry.

Our paper is organised as follows. In Sec. II (with Appendix A), we describe the derivation of the ecKP equation in the context of the classical surface gravity waves problem for an incompressible fluid. From mathematical perspective, the derivation for water wave problems is more challenging than similar derivations for problems where nonlinear and dispersive terms are present in the equations, rather than originating from the free surface boundary conditions. The equation can be readily derived in other physical contexts. In Sec. III, we find transformations between arbitrary solutions of the derived version of the KP equation and the original KP equation. Section IV (with Appendix B) is devoted to the lumps, line solitons, and quasiperiodic solutions of the ecKP-I and ecKP-II equations. In Sec. V, we discuss the approximate solutions for surface waves described by the derived equation. We conclude in Sec. VI by outlining possible applications and generalisations of our results.

\section{DERIVATION OF THE ELLIPTIC CYLINDRICAL KP EQUATION}

We consider the classical water wave problem for an incompressible fluid, described by the full set of Euler equations with free surface and rigid horizontal bottom boundary conditions (1). Since we aim to consider waves with the nearly elliptic front, we write this set of equations in the elliptic cylindrical coordinate system

$$
\begin{aligned}
& x=d \cosh \alpha \cos \beta, \\
& y=d \sinh \alpha \sin \beta, \\
& z=z,
\end{aligned}
$$

where the dimensional parameter $d$ has the meaning of half of the distance between the foci of the coordinate lines, and change the two horizontal components of the velocity vector appropriately

$$
u \rightarrow u \cos \beta-v \sin \beta, \quad v \rightarrow u \sin \beta+v \cos \beta .
$$

Here, we keep the same notations $(u, v, w)$ for the projections of the velocity vector on the new coordinate lines.

Let $z=h_{0}$ be the unperturbed fluid depth, $\lambda$ be the characteristic wavelength, $p_{a}$ the atmospheric pressure, and $h_{s}$ the characteristic free surface elevation. We nondimensionalise the variables

$$
\begin{gathered}
x \rightarrow \lambda x, \quad y \rightarrow \lambda y, \quad z \rightarrow h_{0} z, \quad t \rightarrow \frac{\lambda}{\sqrt{g h_{0}}} t, \\
u \rightarrow \sqrt{g h_{0}} u, \quad v \rightarrow \sqrt{g h_{0}} v, \quad w \rightarrow \frac{h_{0} \sqrt{g h_{0}}}{\lambda} w, \\
h \rightarrow h_{0}+h_{s} \eta, \quad p \rightarrow p_{a}+\rho g\left(h_{0}-z\right)+\rho g h_{0} p,
\end{gathered}
$$

which leads to the appearance of two usual nondimensional parameters in the problem: the long wavelength parameter $\delta=\frac{h_{0}}{\lambda}$, and the small amplitude parameter $\epsilon=\frac{h_{s}}{h_{0}}$, as well as a new nondimensional parameter $\gamma=\frac{d}{\lambda}$, which is not necessarily small. Scaling the dependent variables

$$
u \rightarrow \epsilon u, \quad v \rightarrow \epsilon v, \quad w \rightarrow \epsilon w, \quad p \rightarrow \epsilon p,
$$

we bring the full set of Euler equations in the elliptic cylindrical coordinates to the form

$$
\begin{aligned}
& u_{t}+\epsilon\left[w u_{z}+\frac{E\left(u u_{\alpha}+v u_{\beta}-v^{2}\right)+F\left(v u_{\alpha}-u u_{\beta}+u v\right)}{2 \gamma e^{\alpha} G}\right] \\
& =-\frac{E p_{\alpha}-F p_{\beta}}{2 \gamma e^{\alpha} G}, \\
& v_{t}+\epsilon\left[w v_{z}+\frac{E\left(u v_{\alpha}+v v_{\beta}+u v\right)+F\left(v v_{\alpha}-u v_{\beta}-u^{2}\right)}{2 \gamma e^{\alpha} G}\right] \\
& \quad=-\frac{E p_{\beta}+F p_{\alpha}}{2 \gamma e^{\alpha} G}, \\
& \delta^{2}\left[w_{t}+\epsilon\left\{w w_{z}+\frac{E\left(u w_{\alpha}+v w_{\beta}\right)+F\left(v w_{\alpha}-u w_{\beta}\right)}{2 \gamma e^{\alpha} G}\right\}\right]=-p_{z},
\end{aligned}
$$

$$
w_{z}+\frac{E\left(u_{\alpha}+v_{\beta}+u\right)+F\left(v_{\alpha}-u_{\beta}+v\right)}{2 \gamma e^{\alpha} G}=0,
$$

$$
\begin{aligned}
\left.p\right|_{z=1+\epsilon \eta(\alpha, \beta, t)}= & \eta-W_{e} \delta^{2}\left\{\eta_{\alpha \alpha}+\eta_{\beta \beta}+\epsilon^{2} \frac{\delta^{2}}{\gamma^{2}}\left[\frac{\eta_{\beta}^{2} \eta_{\alpha \alpha}+\eta_{\alpha}^{2} \eta_{\beta \beta}-2 \eta_{\alpha} \eta_{\beta} \eta_{\alpha \beta}}{G}\right.\right. \\
& \left.\left.+\frac{\eta_{\alpha} \sinh 2 \alpha+\eta_{\beta} \sin 2 \beta}{2 G^{2}}\right]\right\} /\left\{\gamma^{2} G\left(1+\epsilon^{2} \frac{\delta^{2}}{\gamma^{2}} \frac{\eta_{\alpha}^{2}+\eta_{\beta}^{2}}{G}\right)^{3 / 2}\right\}
\end{aligned}
$$

$\left.w\right|_{z=1+\epsilon \eta(\alpha, \beta, t)}=\eta_{t}+\epsilon \frac{E\left(u \eta_{\alpha}+v \eta_{\beta}\right)+F\left(v \eta_{\alpha}-u \eta_{\beta}\right)}{G}$,

$$
\left.w\right|_{z=0}=0 .
$$


Here, $W_{e}=\frac{\Gamma}{\rho g h_{0}^{2}}$ is the Weber number, and we denoted

$$
\begin{aligned}
& E=e^{2 \alpha}-\cos 2 \beta, \quad F=\sin 2 \beta, \\
& G=\sinh ^{2} \alpha+\sin ^{2} \beta .
\end{aligned}
$$

This set of equations reduces to the Euler equations written in cylindrical coordinates in the limit

$$
\alpha \rightarrow \infty, \quad \gamma \rightarrow 0 \quad \text { with } \quad \frac{1}{2} \gamma e^{\alpha} \rightarrow r \quad \text { being finite. }
$$

The equation for linear waves (in the long-wave approximation) is easily obtained from Eqs. (7)-(13) with $\epsilon=\delta=0$ as

$$
\eta_{t t}=\frac{\eta_{\alpha \alpha}+\eta_{\beta \beta}}{\gamma^{2}\left(\sinh ^{2} \alpha+\sin ^{2} \beta\right)}
$$

Note that Eq. (15) indeed reduces to the equation

$$
\eta_{t t}-\left(\eta_{r r}+\frac{1}{r} \eta_{r}+\frac{1}{r^{2}} \eta_{\beta \beta}\right)=0
$$

for the long linear waves in the polar cylindrical coordinates in the limit (14). The derivation of the cylindrical KP (cKP) equation (also known as the nearly concentric KP equa$\operatorname{tion}^{3,5}$ ) is based on the existence of solutions of Eq. (16), which do not depend on $\beta$, i.e., there exists an exact reduction of the Eq. (16) to the equation

$$
\eta_{t t}-\left(\eta_{r r}+\frac{1}{r} \eta_{r}\right)=0 .
$$

Unlike Eq. (16), Eq. (15) does not have an exact reduction to the equation with no dependence on $\beta$, which would seem necessary in order to derive a version of the KP equation for waves with nearly elliptic front. Nevertheless, such an equation exists as an asymptotic reduction, and it turns out that this allows for a generalisation of the cKP equation to be derived.

Next, we introduce the variables

$$
\begin{aligned}
\zeta & =\frac{\epsilon^{2}}{\delta^{2}}(\gamma \cosh \alpha-t), \\
R & =\frac{\epsilon^{6}}{\delta^{4}} \gamma \cosh \alpha, \quad \nu=\frac{\delta}{\epsilon^{2}} \sin \beta, \\
u & =\frac{\epsilon^{3}}{\delta^{2}} U, \quad v=\frac{\epsilon^{5}}{\delta^{3}} V, \quad w=\frac{\epsilon^{5}}{\delta^{4}} W, \\
\eta & =\frac{\epsilon^{3}}{\delta^{2}} H, \quad p=\frac{\epsilon^{3}}{\delta^{2}} P,
\end{aligned}
$$

which generalise a change of variables for the cylindrical coordinates. ${ }^{3}$ We use a large distance variable $R$ in preference to large time, but one can also work throughout using an analogous large time variable, $T=\frac{\epsilon^{6}}{\delta^{4}} t$. Here, $2 \gamma \cosh \alpha$ is the nondimensional sum of the distances from a point on an ellipse to its foci. Thus, $\zeta$ is an asymptotic characteristic coordinate for waves with nearly elliptic front, and it becomes the characteristic coordinate for the concentric waves in the limit (14). Note that in this derivation, the variable $\nu$ is proportional to $\sin \beta$ and not just $\beta$, unlike the derivation for the concentric waves. ${ }^{3,5}$ This increases the range of the formal asymptotic validity of the model.

In these variables, the problem formulation (7)-(13) assume the form containing a single small parameter $\Delta=\frac{\epsilon^{4}}{\delta^{2}}$, and a non-dimensional parameter $A=\gamma \frac{\epsilon^{6}}{\delta^{4}}$, which is not necessarily small. The equations are given in Appendix A.

We now seek an asymptotic solution of this system of equations and boundary conditions in the form

$$
H=H_{0}+\Delta H_{1}+O\left(\Delta^{2}\right),
$$

with similar expansions for $U, V, W$, and $P$. At leading order $(O(1))$, we obtain

$$
\begin{aligned}
U_{0 \zeta} & =P_{0 \zeta}, \\
V_{0 \zeta} & =\frac{1}{\sqrt{R^{2}-A^{2}}} P_{0 \nu}+\frac{R-\sqrt{R^{2}-A^{2}}}{\sqrt{R^{2}-A^{2}}} \nu P_{0 \zeta}, \\
P_{0 z} & =0, \quad U_{0 \zeta}+W_{0 z}=0, \\
\left.P_{0}\right|_{z=1} & =H_{0},\left.\quad W_{0}\right|_{z=1}=-H_{0 \zeta},\left.\quad W_{0}\right|_{z=0}=0,
\end{aligned}
$$

which yields, imposing the condition that the perturbation in $U$ is caused only by the passing wave,

$$
\begin{gathered}
P_{0}=H_{0}, \quad U_{0}=H_{0}, \quad W_{0}=-H_{0 \zeta} z \\
V_{0 \zeta}=\frac{1}{\sqrt{R^{2}-A^{2}}} H_{0 \nu}+\frac{R-\sqrt{R^{2}-A^{2}}}{\sqrt{R^{2}-A^{2}}} \nu H_{0 \zeta} .
\end{gathered}
$$

At the next order $(O(\Delta))$, we obtain the following equations and boundary conditions:

$$
\begin{gathered}
U_{1 \zeta}-P_{1 \zeta}=P_{0 R}+U_{0} U_{0 \zeta}+W_{0} U_{0 z} \\
\quad-\frac{R-\sqrt{R^{2}-A^{2}}}{R^{2}-A^{2}}\left(\nu P_{0 \nu}+R \nu^{2} P_{0 \zeta}\right) \\
V_{1 \zeta}-\frac{1}{\sqrt{R^{2}-A^{2}}} P_{1 \nu}-\frac{R-\sqrt{R^{2}-A^{2}}}{\sqrt{R^{2}-A^{2}}} \nu P_{1 \zeta} \\
=U_{0} V_{0 \zeta}+W_{0} V_{0 z}+\frac{R-\sqrt{R^{2}-A^{2}}}{\sqrt{R^{2}-A^{2}}} \nu P_{0 R} \\
+\frac{\left(R-\sqrt{R^{2}-A^{2}}\right)\left(R^{2}+A^{2}\right)}{2\left(R^{2}-A^{2}\right)^{3 / 2} P_{0 \zeta}} \\
-\frac{\left(R-A^{2}-A^{2}\right)^{2}+R^{2}}{2\left(R^{2}-A^{2}\right)^{3 / 2} P_{0 \nu}} \\
P_{1 z}=W_{0 \zeta} \\
U_{1 \zeta}+W_{1 z}=-U_{0 R}+\frac{R-\sqrt{R^{2}-A^{2}}}{R^{2}-A^{2}}\left(R \nu^{2} U_{0 \zeta}+\nu U_{0 \nu}\right) \\
\quad-\frac{1}{\sqrt{R^{2}-A^{2}}}\left(\left(R-\sqrt{R^{2}-A^{2}}\right) \nu V_{0 \zeta}+V_{0 \nu}+U_{0}\right) \\
\left.P_{1}\right|_{z=1}+\left.H_{z=1} P_{0 z}\right|_{z=1}=H_{1}-\left.W_{e} H_{0 z}\right|_{z=1}=-H_{1 \zeta}+U_{0} H_{0 \zeta}
\end{gathered}
$$


Then, Eqs. (17), (21), and (23) yield

$$
P_{1}=-H_{0 \zeta \zeta}\left(\frac{z^{2}-1}{2}+W_{e}\right)+H_{1}
$$

hence from Eqs. (19), (22), and (25), we find, using Eq. (17), that

$$
\begin{aligned}
W_{1}= & H_{0 \zeta \zeta \zeta}\left[\frac{z^{3}}{6}+\left(W_{e}-\frac{1}{2}\right) z\right] \\
& -\left[H_{1 \zeta}+2 H_{0 R}+H_{0} H_{0 \zeta}-\frac{R-\sqrt{R^{2}-A^{2}}}{R^{2}-A^{2}}\left(2 \nu H_{0 \nu}\right.\right. \\
& \left.+2 R \nu^{2} H_{0 \zeta}\right)+\frac{1}{\sqrt{R^{2}-A^{2}}}\left(\left(R-\sqrt{R^{2}-A^{2}}\right) \nu V_{0 \zeta}\right. \\
& \left.\left.+V_{0 \nu}+H_{0}\right)\right] z .
\end{aligned}
$$

Finally, substituting $W_{1}$ into the remaining boundary condition (24), differentiating with respect to $\zeta$ and using Eq. (18), we obtain ecKP equation

$$
\begin{aligned}
& {\left[2 H_{0 R}+3 H_{0} H_{0 \zeta}+\left(\frac{1}{3}-W_{e}\right) H_{0 \zeta \zeta \zeta}+\frac{R}{R^{2}-A^{2}} H_{0}\right.} \\
& \left.-A^{2} \frac{\nu^{2}}{R^{2}-A^{2}} H_{0 \zeta}\right]_{\zeta}+\frac{1}{R^{2}-A^{2}} H_{0 \nu \nu}=0 .
\end{aligned}
$$

Note that Eq. (20), written for the completeness of the set of equations, allows one to find $V_{1}$ and is not used in the derivation of the ecKP equation.

The scaling transformation

$$
\widetilde{\alpha} R \rightarrow \tau, \quad \widetilde{\beta} \zeta \rightarrow \zeta, \quad \widetilde{\gamma} \nu \rightarrow \nu, \quad H_{0} \rightarrow \widetilde{\delta} H
$$

where $\widetilde{\alpha}$ is a free parameter and

$$
\begin{aligned}
\widetilde{\beta} & =\left(\frac{2 \widetilde{\alpha}}{\frac{1}{3}-W_{e}}\right)^{1 / 3}, \\
\widetilde{\gamma} & =\left(\sigma \widetilde{\alpha} \widetilde{\beta} \sigma^{2}\right)^{1 / 2}, \\
\widetilde{\delta} & =4 \frac{\widetilde{\alpha}}{\widetilde{\beta}}, \\
\sigma^{2} & =\operatorname{sign}(\widetilde{\alpha} \widetilde{\beta})
\end{aligned}
$$

brings the derived Eq. (27) to the form

$$
\begin{aligned}
& \left(H_{\tau}+6 H H_{\zeta}+H_{\zeta \zeta \zeta}+\frac{\tau}{2\left(\tau^{2}-a^{2}\right)} H\right. \\
& \left.\quad-\frac{a^{2} \nu^{2}}{12 \widetilde{\alpha}^{2} \sigma^{2}\left(\tau^{2}-a^{2}\right)} H_{\zeta}\right)_{\zeta}+\frac{3 \sigma^{2}}{\tau^{2}-a^{2}} H_{\nu \nu}=0,
\end{aligned}
$$

shown in the Introduction. Here, $a=\widetilde{\alpha} A$. If we let $\widetilde{\alpha}=1$, then $a=A$ and $\sigma^{2}=\operatorname{sign}\left(\frac{1}{3}-W_{e}\right)$. For typical water waves, $\sigma^{2}=1\left(W_{e}<\frac{1}{3}\right)$. However, $\sigma^{2}=-1$ if the effects of surface tension are strong $\left(W_{e}>\frac{1}{3}\right)$. It is natural to call the corresponding equations ecKP-II and ecKP-I, respectively, similarly to the terminology used in the Cartesian geometry.

\section{TRANSFORMATIONS BETWEEN KP, CKP, AND ECKP EQUATIONS}

Considerations used to find the mapping from the solutions of the $\mathrm{KdV}$ equation to the class of solutions of the cKP equation ${ }^{3,5}$ can be extended to obtain transformations between arbitrary solutions of all three versions of the KP equation, related to the Cartesian, cylindrical, and elliptic cylindrical coordinates, respectively. The resulting transformations generalise the transformations between the KP and cKP equations, ${ }^{3,5,7}$ discussed in the Introduction.

Indeed, the geometry of a wave with nearly elliptic front, considered simultaneously in the Cartesian and elliptic cylindrical coordinates, suggests the introduction of the sum and the difference of the nondimensional distances from a point on the wave front to the two foci of the coordinate system

$$
\begin{gathered}
d_{1}+d_{2}=2 \gamma \cosh \alpha, \\
d_{1}-d_{2}=2 \gamma \cos \beta,
\end{gathered}
$$

where the foci have the following Cartesian coordinates: $F_{1}(-\gamma, 0)$ and $F_{2}(\gamma, 0)$. We recall that the variables have been nondimensionalised, as discussed in Sec. II, and $\gamma=\frac{d}{2}$. Note that $\frac{1}{2}\left(d_{1}+d_{2}\right)-t$ corresponds, up to the scaling, to the asymptotic characteristic variable $\zeta$, introduced in Sec. II.

Then, for the area satisfying $\frac{y}{x-\gamma}, \frac{y}{x+\gamma} \rightarrow 0$, we obtain the following asymptotic behaviour:

$$
\begin{aligned}
\frac{1}{2}\left(d_{1}+d_{2}\right)-t= & \frac{1}{2}\left(\sqrt{(x+\gamma)^{2}+y^{2}}+\sqrt{(x-\gamma)^{2}+y^{2}}\right)-t \\
& \sim x-t+\frac{1}{4} y^{2}\left(\frac{1}{x+\gamma}+\frac{1}{x-\gamma}\right) .
\end{aligned}
$$

Next, for sufficiently large $\alpha$ and small $\beta$, our nondimensional variable $x=\gamma \cosh \alpha \cos \beta \sim \frac{\delta^{4}}{\epsilon^{6}} R$, and the previous asymptotics can be rewritten as

$$
\frac{1}{2}\left(d_{1}+d_{2}\right)-t \sim \xi+\frac{1}{2} Y^{2} \frac{R}{R^{2}-A^{2}},
$$

where $\xi=x-t, Y=\frac{\epsilon^{3}}{\delta^{2}} y$, and $A=\gamma \frac{\epsilon^{6}}{\delta^{4}}$. Similarly,

$$
\frac{1}{2}\left(d_{1}-d_{2}\right) \sim \gamma-\frac{A}{2} \frac{Y^{2}}{R^{2}-A^{2}} .
$$

This asymptotic behaviour of the geometrically meaningful objects motivates the change of variables

$$
H_{0}(R, \zeta, \nu)=\eta(R, \xi, Y),
$$

where

$$
\zeta=\xi+\frac{1}{2} Y^{2} \frac{R}{R^{2}-A^{2}}, \quad \nu=\frac{Y}{\sqrt{R^{2}-A^{2}}} .
$$

It is then verified by direct calculation that this transformation maps the ecKP Eq. (27) to the KP equation, written in the form

$$
\left[2 \eta_{R}+3 \eta \eta_{\xi}+\left(\frac{1}{3}-W_{e}\right) \eta_{\xi \xi \xi}\right]_{\xi}+\eta_{Y Y}=0 .
$$


To finish this section, let us summarise the transformations between all three versions of the KP equation. We write the KP equation in the canonical form

$$
\left(U_{\tau}+6 U U_{\xi}+U_{\xi \xi \xi}\right)_{\xi}+3 \sigma^{2} U_{Y Y}=0,
$$

the cKP equation in the similar form

$$
\left(W_{\tau}+6 W W_{\chi}+W_{\chi \chi \chi}+\frac{1}{2 \tau} W\right)_{\chi}+\frac{3 \sigma^{2}}{\tau^{2}} W_{V V}=0,
$$

and the ecKP equation as

$$
\begin{aligned}
& \left(H_{\tau}+6 H H_{\zeta}+H_{\zeta \zeta \zeta}+\frac{\tau}{2\left(\tau^{2}-a^{2}\right)} H-\frac{a^{2} \nu^{2}}{12 \sigma^{2}\left(\tau^{2}-a^{2}\right)} H_{\zeta}\right)_{\zeta} \\
& +\frac{3 \sigma^{2}}{\tau^{2}-a^{2}} H_{\nu \nu}=0 .
\end{aligned}
$$

Then, the map

$$
U(\tau, \xi, Y) \rightarrow W(\tau, \chi, V):=U\left(\tau, \chi-\frac{\tau V^{2}}{12 \sigma^{2}}, \tau V\right)
$$

transforms any solution of the KP equation into a solution of the cKP equation, and the map

$$
U(\tau, \xi, Y) \rightarrow H(\tau, \zeta, \nu):=U\left(\tau, \zeta-\frac{\tau \nu^{2}}{12 \sigma^{2}}, \sqrt{\tau^{2}-a^{2}} \nu\right)
$$

transforms any solution of the KP equation into a solution of the ecKP equation. Note that the second transformation reduces to the first in the limit $a \rightarrow 0$. The map (30) also shows that for small $a$ and small values of $\tau$, any solution of the ecKP equation approaches some $Y$-independent solution of the KP equation. These transformations can be inverted, and they can also be used to obtain the direct transformations between the cKP and ecKP equations.

Indeed, the map inverting (30) has the form

$$
\begin{aligned}
& H(\tau, \zeta, \nu) \rightarrow \\
& U(\tau, \xi, Y):=H\left(\tau, \xi+\frac{1}{12 \sigma^{2}} \frac{\tau Y^{2}}{\tau^{2}-a^{2}}, \frac{Y}{\sqrt{\tau^{2}-a^{2}}}\right) .
\end{aligned}
$$

It transforms any solution of the ecKP equation into a solution of the KP equation. In particular, this map shows that for very large values of $\tau$ and finite values of $Y$, any solution of the ecKP equation will approach a $Y$-independent solution of the KP equation (possibly, a constant or zero). However, such large values of $\tau$ are likely to lie outside of the range of applicability of the derived model, and we do not discuss this limit any more.

The map

$$
\begin{aligned}
& W(\tau, \chi, V) \rightarrow \\
& H(\tau, \zeta, \nu):=W\left(\tau, \zeta-\frac{a^{2} \nu^{2}}{12 \sigma^{2} \tau}, \frac{\sqrt{\tau^{2}-a^{2}}}{\tau} \nu\right)
\end{aligned}
$$

transforms any solution of the cKP equation into a solution of the ecKP equation, and the map

$$
\begin{aligned}
& H(\tau, \zeta, \nu) \rightarrow \\
& W(\tau, \chi, V):=H\left(\tau, \chi+\frac{a^{2} \tau V^{2}}{12 \sigma^{2}\left(\tau^{2}-a^{2}\right)}, \frac{\tau}{\sqrt{\tau^{2}-a^{2}}} V\right)
\end{aligned}
$$

transforms any solution of the ecKP equation into a solution of the cKP equation.

\section{SPECIAL SOLUTIONS OF ECKP-I AND ecKP-II EQUATIONS}

In this section, we will consider some special solutions to the ecKP Eq. (29) per se, to illustrate the characteristic features of the equation. The considered examples are exact solutions to the KP-I and KP-II equations: lumps, line solitons, and quasiperiodic solutions (see, Refs. 21 and 22), which become solutions to the ecKP-I and ecKP-II equations under the map (30).

If the $a$ in Eq. (30) vanishes, the ecKP solution reduces to the corresponding cKP solution. For small "times" $\tau$, the cKP solutions look like solutions to the $\mathrm{KdV}$ equation (essentially no dependence on the transversal variable), whereas they develop horseshoe-type profiles for larger $\tau$. The ecKP solutions on the other hand show such profiles already for small $\tau-a$ if $a>0$. For large $\tau(\tau \gg a)$, the solutions tend asymptotically
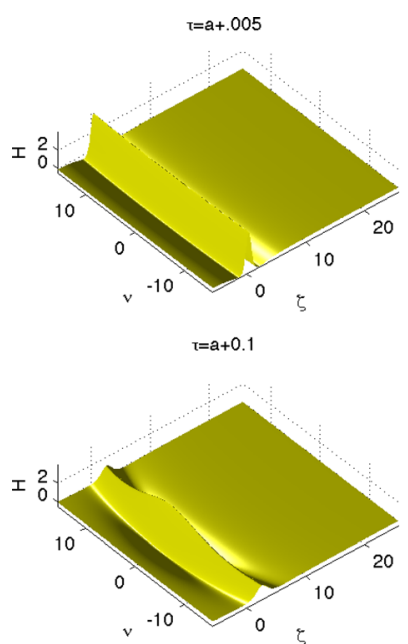

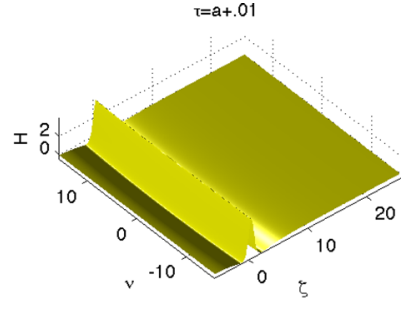

$\tau=a+1$

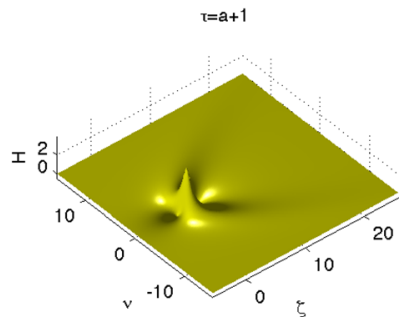

FIG. 1. Solution to the ecKP-I equation obtained as the image of the lump (31) with $\kappa=1$ under the action of the map (30) for $a=0.01$ and several values of $\tau$.
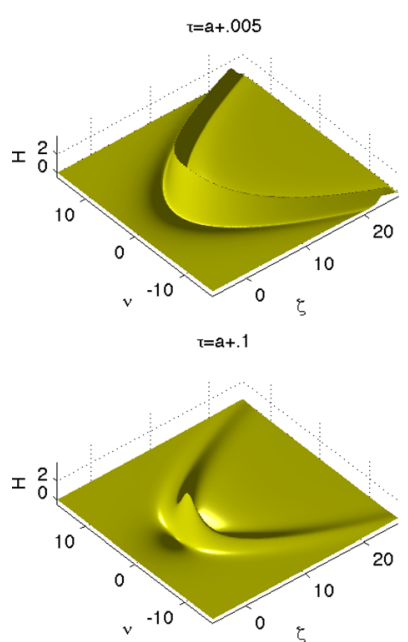
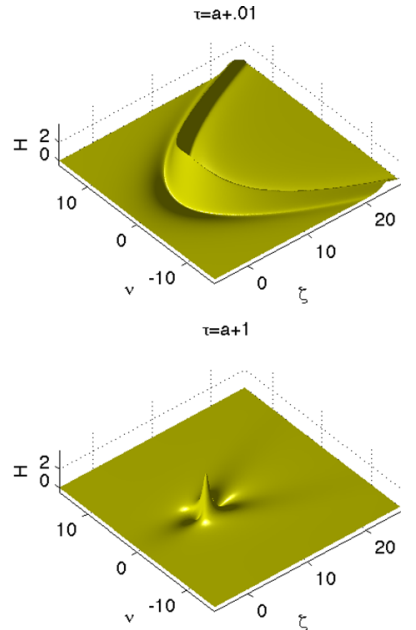

FIG. 2. Solution to the ecKP-I equation obtained as the image of the lump (31) with $\kappa=1$ under the action of the map (30) for $a=1$ and several values of $\tau$. 

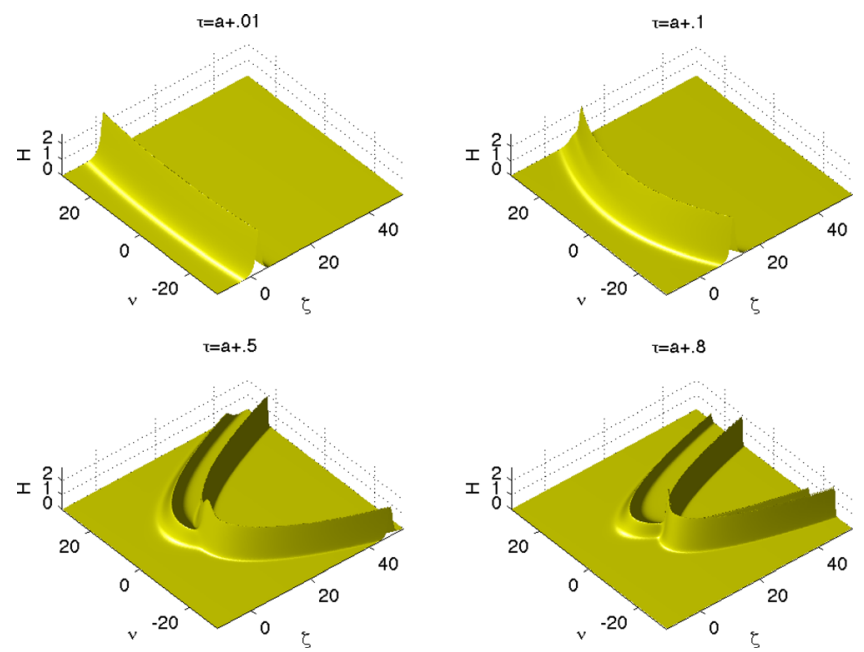

FIG. 3. 2-soliton solution of the ecKP-II equation for $a=0.01$ with $k_{1}=1.5$, $k_{2}=0.5, k_{3}=-2$, and $k_{4}=0$ for several values of $\tau$.

to the corresponding cKP solutions. We will illustrate this behaviour at several examples.

The first example we consider is the KP-I lump solution,

$$
U(\xi, Y, \tau)=\frac{4 \kappa\left(1-\kappa(\xi-3 \kappa \tau)^{2}+\kappa^{2} Y^{2}\right)}{\left(1+\kappa(\xi-3 \kappa \tau)^{2}+\kappa^{2} Y^{2}\right)^{2}},
$$

with $\kappa=1$, under the map (30). It is visible that when $\tau \rightarrow a$ and $a$ are close to zero, the solution $H(\zeta, \nu, \tau)$ is essentially independent of the coordinate $\nu$. This can be seen for $a=0.01$ in Fig. 1. For larger values of $a$, the solution has a parabolic shape for small $\tau-a$ as can be seen in Fig. 2 .

Next, we consider the 2-soliton solution of the KP-II equation in the form

$$
U(\xi, Y, \tau)=2 \partial_{x}^{2} \ln W\left(e^{\vartheta_{1}}+e^{\vartheta_{2}}, e^{\vartheta_{3}}+e^{\vartheta_{4}}\right),
$$

where $\theta_{j}=k_{j} \xi+k_{j}^{2} Y-4 k_{j}^{3} \tau, k_{j}$ are arbitrary constants, and $W$ is the Wronskian of the two functions. It can be seen for $a=0.01$, i.e., close to the cKP case, in Fig. 3 where the formation of horseshoe waves can be clearly recognised. The
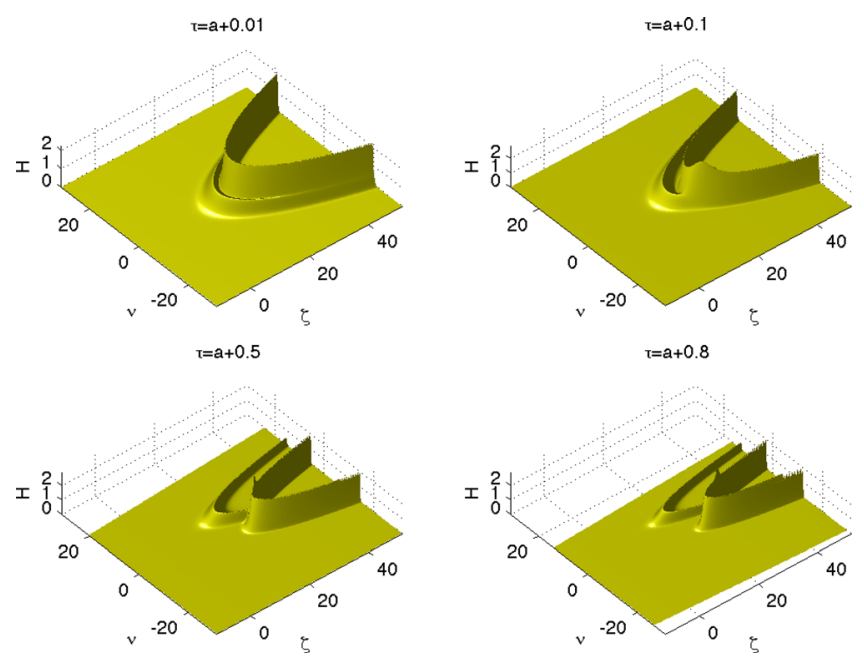

FIG. 4. 2-soliton solution of the ecKP-II equation for $a=1$ with $k_{1}=1.5$, $k_{2}=0.5, k_{3}=-2$, and $k_{4}=0$ for several values of $\tau$. corresponding ecKP solution for $a=1$ is shown in Fig. 4 where the curved profiles are already present for small $\tau-a$.

Quasi-periodic (multiphase) solutions of the ecKP equation can be obtained as the image of the known thetafunctional solutions of the KP equation under the map (30). The solutions are shown in Appendix B.

While the solutions of the ecKP equation are qualitatively similar to the solutions of the cKP equation, significant differences can be seen at the level of approximate solutions for the Euler equations, as shown in Sec. V.

\section{APPROXIMATE SOLUTIONS FOR SURFACE WAVES}

Exact solutions of the derived equation allow us to obtain new asymptotic solutions for the classical water wave problem (1). In order to do that we return to the original nondimensional variables $x, y, t$ and re-parametrise our solution as follows:

$$
\begin{gathered}
x=\gamma \cosh \alpha \cos \beta, \quad y=\gamma \sinh \alpha \sin \beta, \\
\eta=\frac{4}{6^{1 / 3}}\left(1-3 W_{e}\right)^{1 / 3} \sqrt{\frac{a}{\gamma}} H(\tau, \zeta, \nu),
\end{gathered}
$$

where

$$
\begin{gathered}
\left(H_{\tau}+6 H H_{\zeta}+H_{\zeta \zeta \zeta}+\frac{\tau}{2\left(\tau^{2}-a^{2}\right)} H-\frac{a^{2} \nu^{2}}{12 \sigma^{2}\left(\tau^{2}-a^{2}\right)} H_{\zeta}\right)_{\zeta} \\
+\frac{3 \sigma^{2}}{\tau^{2}-a^{2}} H_{\nu \nu}=0, \\
\sigma^{2}=\operatorname{sign}\left(1-3 W_{e}\right) \quad \text { and } \quad \tau=R=a \cosh \alpha, \\
\zeta=\frac{6^{1 / 3} a}{\gamma \Delta\left(1-3 W_{e}\right)^{1 / 3}}(\gamma \cosh \alpha-t), \\
\nu=\frac{6^{2 / 3}}{\Delta^{1 / 2}\left|1-3 W_{e}\right|^{1 / 6}} \sin \beta .
\end{gathered}
$$

Here, $t$ is the physical time (nondimensional). Below, we assume that $t \geq 0$ and consider the initial stages of the evolution. We also have $\epsilon=\sqrt{\frac{\gamma}{a}} \Delta$ and $\delta=\frac{\gamma}{a} \Delta^{3 / 2}$. Since asymptotic longwave models usually provide a good qualitative (often quantitative) description even outside of the range of their formal asymptotic validity (i.e., the physical applicability of such models is usually wider than their formal asymptotic validity), we plot the solutions for all $0 \leq \beta<2 \pi$ and $\alpha \geq 0$. Unless it is explicitly stated otherwise, at least parts of the shown solutions belong to the range of the formal validity of the asymptotic model (defined by $\tau \sim O(1), \zeta \sim O(1)$, and $\nu \sim O(1)$ as $\Delta \rightarrow 0$ ).

The 1-soliton solution of the ecKP-II equation (i.e., the image of the 1-soliton solution of the KP-II equation under the map (30)) is explicitly written in the form

$$
\begin{aligned}
H(\tau, \zeta, \nu)= & \frac{K^{2}}{2} \operatorname{sech}^{2} \\
& {\left[\frac{K}{2}\left(\zeta-\frac{\tau \nu^{2}}{12}+L \sqrt{\tau^{2}-a^{2}} \nu-\left(K^{2}+3 L^{2}\right) \tau+\delta_{0}\right)\right], }
\end{aligned}
$$

where $K, L$, and $\delta_{0}$ are arbitrary constants. In the examples shown below, we let the Weber number $W_{e}=0$ and the phase shift $\delta_{0}=0$. It turns out that this single formula 

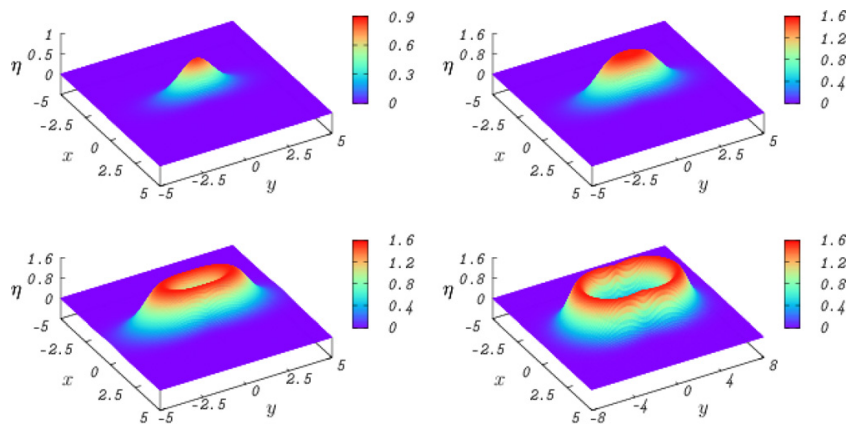

FIG. 5. Surface wave corresponding to the one-soliton solution (34) of the ecKP-II equation with $K=1$ and $L=0$ for $t=0$ (top left), $t=0.25$ (top right), $t=0.5$ (bottom left), and $t=1$ (bottom right).

describes a variety of wave fronts. In what follows we provide the complete classification of these wave fronts, obtaining characteristic conditions on the parameters of the solutions (34) distinguishing various cases. We illustrate most of the wave fronts, plotting the corresponding surface wave elevation $\eta$ for $\gamma=1, a=2$, and $\Delta=1 / 2$. Similar solutions exist for the ecKP-I equation, as the image of an (unstable) line-soliton of the KP-I equation. We also note that an analogue of the solution (34) in cylindrical geometry (cKP) describes only a single type of a wave front (the picture is qualitatively similar to a part of the wave front shown in Fig. 5 above), and it can be plotted only for the limited values of the polar angle (even formally).

The wave obtained when $K=1$ and $L=0$ is compact and symmetric, it is shown in Fig. 5 above.

For $L \neq 0$, the solution has no symmetry with respect to the $y$-coordinate, as one can see in Fig. 6 for $K=1$ and $L=0.1$. The change $L \rightarrow-L$ yields the reflection of the wave front with respect to the $x$-axis

$$
\eta(-L, x, y)=\eta(L, x,-y)
$$

Therefore, it suffices to consider $L>0$ or $L<0$.

When $|L|$ increases further, the compact nearly elliptic wave shape disappears. The wave becomes non-compact, and it rather describes the deformed line soliton, featuring an elliptic inhomogeneity in the central part of the wave. The solution is shown in Fig. 7 for $K=1$ and $L=-0.5$.

For sufficiently large $|L|$ solution is localised in the vicinity of some point satisfying the relation $L \sin \beta=|L|$ and
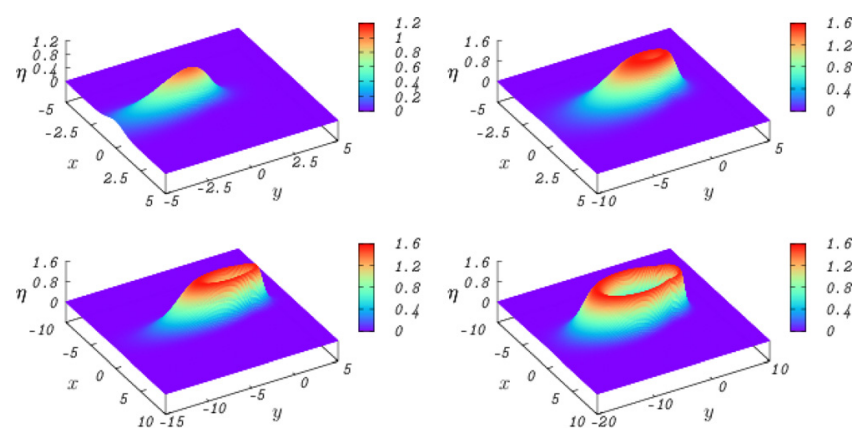

FIG. 6. Surface wave corresponding to the one-soliton solution (34) of the ecKP-II equation with $K=1$ and $L=0.1$ for $t=0$ (top left), $t=0.25$ (top right), $t=0.5$ (bottom left), and $t=1$ (bottom right).
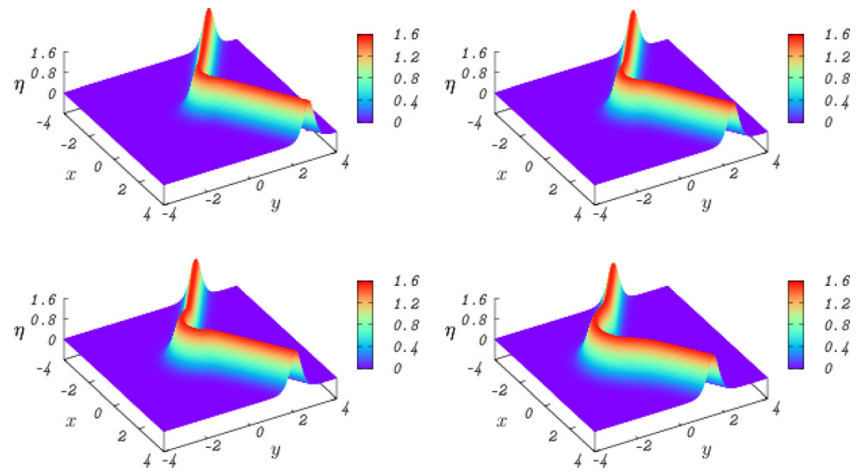

FIG. 7. Surface wave corresponding to the one-soliton solution (34) of the ecKP-II equation with $K=1$ and $L=-0.5$ for $t=0$ (top left), $t=0.25$ (top right), $t=0.5$ (bottom left), and $t=2$ (bottom right).

strongly attenuates with time. (The large values of $L$ lie outside of the range of validity of the model.)

In order to explain the observed features of the solution (34) and to obtain the corresponding conditions for the parameters of the solution, let us note that the maximum of its amplitude is attained when the argument of $\operatorname{sech}^{2}$ is equal to zero (if this is possible)

$$
\zeta-\frac{\tau \nu^{2}}{12}+L \sqrt{\tau^{2}-a^{2}} \nu-\left(K^{2}+3 L^{2}\right) \tau=0 .
$$

This condition can be written either as

$$
\begin{aligned}
& \left(2-\sin ^{2} \beta-\frac{2 \Delta}{6^{1 / 3}}\left[K^{2}+3 L^{2}\right]\right) \cosh \alpha \\
& +2 \Delta^{1 / 2} 6^{1 / 3} L \sin \beta \sinh \alpha-\frac{2 t}{\gamma}=0
\end{aligned}
$$

or as

$$
\begin{aligned}
& \sin ^{2} \beta-2 \Delta^{1 / 2} 6^{1 / 3} L \tanh \alpha \sin \beta \\
& +\frac{2 \Delta}{6^{1 / 3}}\left[K^{2}+3 L^{2}\right]-2+\frac{2 t}{\gamma} \operatorname{sech} \alpha=0 .
\end{aligned}
$$

Let us first consider the case $L=0$. For

$$
K^{2} \leq \frac{6^{1 / 3}}{2 \Delta}
$$

the solution has the form of a compact nearly elliptic wave of narrowing width (as shown in Fig. 5). Indeed,

$$
1-\frac{2 \Delta}{6^{1 / 3}} K^{2} \leq \frac{2 t}{\gamma} \operatorname{sech} \alpha \leq 2-\frac{2 \Delta}{6^{1 / 3}} K^{2} .
$$

We note that although the width of the wave clearly changes, the amplitude is constant, which can be viewed as the manifestation of the solitonic nature of this solution.

For

$$
\frac{6^{1 / 3}}{2 \Delta} \leq K^{2} \leq \frac{6^{1 / 3}}{\Delta}\left(\approx 3.63 \text { for } \Delta=\frac{1}{2}\right)
$$

the condition (37) is satisfied for 


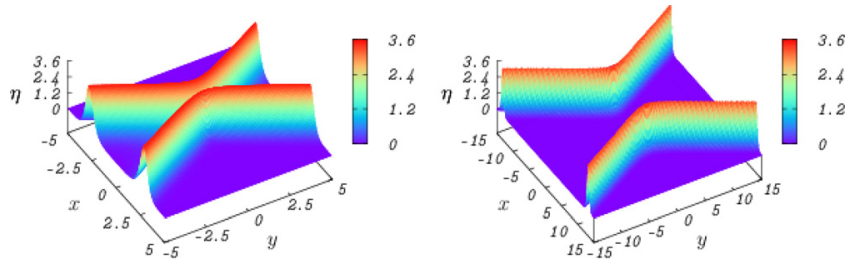

FIG. 8. Surface waves corresponding to the one-soliton solution of the ecKP-II Eq. (34) with $K=1.5$ and $L=0$ for $t=0$ (left) and $t=2$ (right).

$$
|\sin \beta|=\sqrt{2-\frac{2 \Delta}{6^{1 / 3}} K^{2}-\frac{2 t}{\gamma} \operatorname{sech} \alpha,}
$$

and the wave splits into two deformed line solitons (shown in Fig. 8).

If

$$
K^{2}>\frac{6^{1 / 3}}{\Delta}
$$

then the argument of Eq. (34) cannot be equal to zero, and the wave continuously attenuates with time. (The large values of $K$ lie outside of the range of validity of the model.)

Similarly, we can consider the case $L \neq 0$ (without loss of generality, we assume that $L>0$, see Eq. (35)). If

$$
\frac{\Delta}{6^{1 / 3}}\left[K^{2}+3 L^{2}\right]>1
$$

then the argument (36) of $\operatorname{sech}^{2}$ is negative for $L \sin \beta<0$ already at $t=0$, and it decreases with the increase of $t$. For $L \sin \beta=|L|$, the argument is closer to zero than for other values of $\beta$. Therefore, $\eta$ has a maximum for $L \sin \beta=|L|$ as a function of $\beta$. However, as we mentioned above, such large values of $K$ and $L$ are likely to be outside of the range of validity of the model, we mention them here only for the completeness of our analysis.

For sufficiently small $K$ and $L$, Eq. (37) yields

$$
\sin \beta=\Delta^{1 / 2} 6^{1 / 3} L \tanh \alpha \pm \sqrt{D}
$$

where

$$
D=2-\frac{2 \Delta}{6^{1 / 3}} K^{2}-\Delta 6^{1 / 3} L^{2} \operatorname{sech}^{2} \alpha-\frac{2 t}{\gamma} \operatorname{sech} \alpha .
$$

Then, for $\alpha \rightarrow \infty$, we obtain

$$
\sin \beta=\Delta^{1 / 2} 6^{1 / 3} L \pm \sqrt{2-\frac{2 \Delta}{6^{1 / 3}} K^{2}}
$$

Therefore, there exist several natural domains for the values of the parameters $K$ and $L$. Let us note that if $2-\frac{2 \Delta}{6^{1 / 3}} K^{2}<0$, then the solution (34) continuously attenuates, and we do not consider this range of values. Let $2-\frac{2 \Delta}{6^{1 / 3}} K^{2}>0$. Then, there are several cases.

(i) If $\left|\Delta^{1 / 2} 6^{1 / 3} L \pm \sqrt{2-\frac{2 \Delta}{6^{1 / 3}} K^{2}}\right|>1$, the solution (34) has the form of a deformed nearly elliptic wave (shown in
Fig. 6). Indeed, in this case, the argument of solution (34) cannot be equal to zero for sufficiently large $\alpha$, and, therefore, the solution is localised. For sufficiently small $L$, the above inequality implies

$$
\begin{aligned}
& \Delta^{1 / 2} 6^{1 / 3} L+\sqrt{2-\frac{2 \Delta}{6^{1 / 3}} K^{2}}>1 \text { and } \\
& \Delta^{1 / 2} 6^{1 / 3} L-\sqrt{2-\frac{2 \Delta}{6^{1 / 3}} K^{2}}<-1,
\end{aligned}
$$

which yields

$$
\sqrt{2-\frac{2 \Delta}{6^{1 / 3}} K^{2}}>1+\Delta^{1 / 2} 6^{1 / 3} L
$$

and

$$
K^{2}+3 L^{2}+\frac{6^{2 / 3}}{\Delta^{1 / 2}} L<\frac{6^{1 / 3}}{2 \Delta}
$$

It is easy to see that if inequality (38) holds, then for any $\beta$

$$
2-\sin ^{2} \beta-\frac{2 \Delta}{6^{1 / 3}}\left[K^{2}+3 L^{2}\right]>2 \Delta^{1 / 2} 6^{1 / 3} L|\sin \beta| .
$$

Therefore, we can introduce the notations

$$
\begin{aligned}
2-\sin ^{2} \beta-\frac{2 \Delta}{6^{1 / 3}}\left[K^{2}+3 L^{2}\right] & =A(\beta) \cosh \left(\alpha_{0}\right), \\
2 \Delta^{1 / 2} 6^{1 / 3} L \sin \beta & =A(\beta) \sinh \left(\alpha_{0}\right),
\end{aligned}
$$

where

$$
\begin{gathered}
A(\beta)=\left\{\left(2-\sin ^{2} \beta-\frac{2 \Delta}{6^{1 / 3}}\left[K^{2}+3 L^{2}\right]\right)^{2}\right. \\
\left.-4 \Delta 6^{2 / 3} L^{2} \sin ^{2} \beta\right\}^{1 / 2}, \\
\tanh \alpha_{0}=\frac{2 \Delta^{1 / 2} 6^{1 / 3} L \sin \beta}{2-\sin ^{2} \beta-\frac{2 \Delta}{6^{1 / 3}}\left[K^{2}+3 L^{2}\right]} .
\end{gathered}
$$

In these notations, Eq. (36) takes the form

$$
\cosh \left(\alpha+\alpha_{0}\right)=\frac{2 t}{A(\beta) \gamma} .
$$

Therefore, in this case, for any $\beta$ and for sufficiently large $t$, there exists the value of the parameter $\alpha$ such that the argument (36) will be equal to zero. Because all functions are continuous and differentiable, the maximum of $H(\tau, \zeta, \nu)$ will be attained along some smooth closed curve. For sufficiently small $t$ and for any $\alpha, \beta$, the value of the function $H(\tau, \zeta, \nu)$ will be less than $K^{2} / 2$. Since the sign of $\alpha_{0}$ depends on the sign of $\beta$, for $L \neq 0$, it follows that the solution will be asymmetric with respect to $\beta$. For $L \sin \beta<0$, the wave will be wider than for $L \sin \beta>0$ (shown in Fig. 6). 

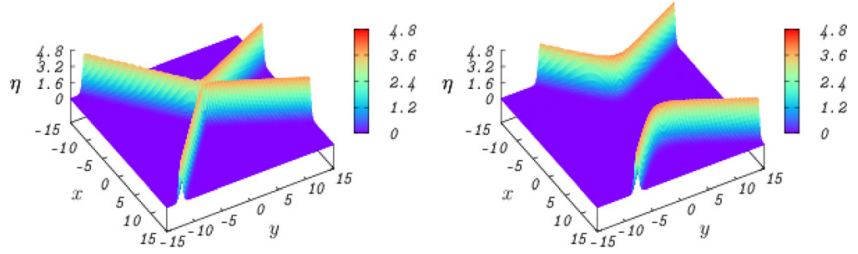

FIG. 9. Surface waves corresponding to the one-soliton solution of the ecKP-II Eq. (34) with $K=1.6$ and $L=0.1$ for $t=0$ (left) and $t=2$ (right).

(ii) If $\Delta^{1 / 2} 6^{1 / 3} L+\sqrt{2-\frac{2 \Delta}{6^{1 / 3}} K^{2}}>1$ and $1>\Delta^{1 / 2} 6^{1 / 3} L$ $-\sqrt{2-\frac{2 \Delta}{6^{1 / 3}} K^{2}}>-1$, the nearly elliptic wave breaks for negative $y$, producing a single deformed line-soliton (shown in Fig. 7).

(iii) If $\left|\Delta^{1 / 2} 6^{1 / 3} L \pm \sqrt{2-\frac{2 \Delta}{6^{1 / 3}} K^{2}}\right|<1$, the nearly elliptic wave breaks both for negative and positive $y$, producing a pair of deformed line-solitons (shown in Fig. 9).

Apart from these generic cases, there are also some exceptional cases (corresponding to the boundaries between the generic cases). To illustrate that we show a solution corresponding to the condition

$$
\Delta^{1 / 2} 6^{1 / 3} L+\sqrt{2-\frac{2 \Delta}{6^{1 / 3}} K^{2}}=1,
$$

which is the borderline case in between the last two generic cases. The solution is shown in Fig. 10, and it can be interpreted as describing the splitting of the wave looking like a resonant Y-soliton (or "Miles soliton" ${ }^{23}$ ), known from the theory of the original KP equation, into two deformed line solitons. The existence of such solutions might indicate the instability of the Y-soliton with respect to perturbations in the area of the crossing.

Let us note that the one-soliton solution of the KP-II equation used to obtain the solution (34) of the ecKP-II equation above can be written in the form of the "canonical" soliton for the KP-II equation

$$
\begin{gathered}
U(\tau, \xi, Y)=2 \partial_{\xi}^{2} \ln \left(\psi_{1}+\psi_{2}\right), \quad \text { where } \\
\psi_{j}=\exp \left(k_{j} \xi+\sigma k_{j}^{2} Y-4 k_{j}^{3} \tau\right),
\end{gathered}
$$

if $K=k_{1}-k_{2}$ and $L=\left(k_{1}+k_{2}\right) \sigma$ (we let $\delta_{0}=0$ ). Then, the conditions on $k_{j}$ follow from the conditions on $K$ and $L$, discussed above. We show in Fig. 11 the surface wave corresponding to the solution of ecKP-II equation obtained as the image of the canonical one-soliton solution of KP-II equation with $\sigma=1, k_{1}=0.5$, and $k_{2}=-0.4$ under the map (30).

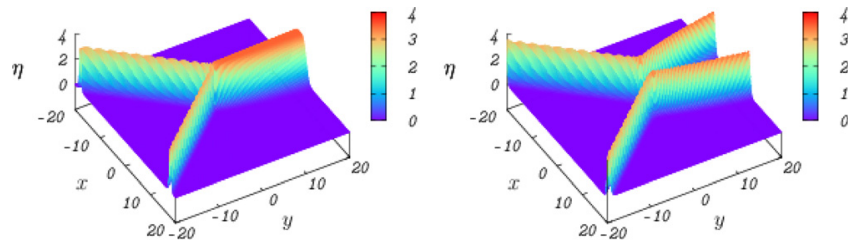

FIG. 10. Surface waves corresponding to the exceptional one-soliton solution of the ecKP-II Eq. (34) with $K=1.5$ and $L$ defined by Eq. (40) $(L \approx 0.1)$ for $t=0$ (left) and $t=1$ (right).

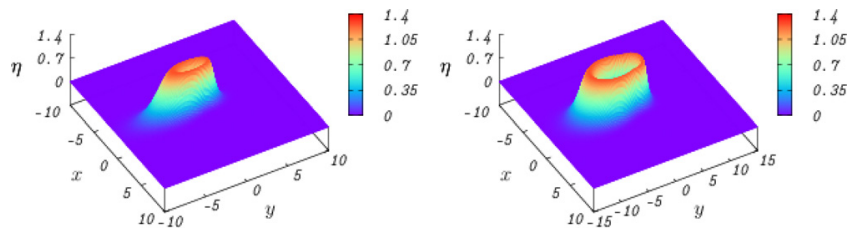

FIG. 11. Surface wave corresponding to the solution of the ecKP-II equation obtained from the canonical KP-soliton with $k_{1}=0.5$ and $k_{2}=-0.4$ for $t=0.5$ (left) and $t=1$ (right).

Using Darboux transformations, one can obtain the canonical two-soliton solution of the KP-II equation in the form

$$
\begin{gathered}
U(\tau, \xi, Y)=2 \partial_{\xi}^{2} \ln \left(\phi_{1} \phi_{2 \xi}-\phi_{2} \phi_{1 \xi}\right), \quad \text { where } \\
\phi_{1}=\psi_{1}-\psi_{2}, \quad \phi_{2}=\psi_{3}+\psi_{4}
\end{gathered}
$$

(up to the phase shifts, which can be added to the phases). Some particular surface waves corresponding to the twosoliton solutions of the ecKP-II equation (obtained as the image of solution (41) under the map (30)) are shown in Figs. 12 and 13 (asymmetric and symmetric two-soliton nearly elliptic waves, respectively).

Finally, let us choose $W_{e}=2 / 3$ and consider the ecKP-I lump solution (the image of the KP-I lump under the map (30))

$$
H(\tau, \zeta, \nu)=\frac{4 \kappa\left(1-\kappa\left(\zeta+\tau \nu^{2} / 12-3 \kappa \tau\right)^{2}+\kappa^{2}\left(\tau^{2}-a^{2}\right) \nu^{2}\right)}{\left(1+\kappa\left(\zeta+\tau \nu^{2} / 12-3 \kappa \tau\right)^{2}+\kappa^{2}\left(\tau^{2}-a^{2}\right) \nu^{2}\right)^{2}} .
$$
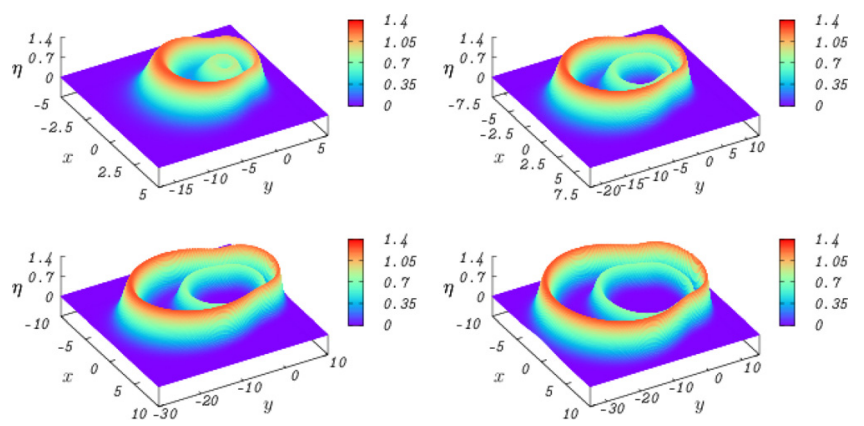

FIG. 12. Surface waves corresponding to the two-soliton solution of the ecKP-II equation with $k_{1}=0.5, k_{2}=-0.4, k_{3}=0.4$, and $k_{4}=-0.3$ for $t=1$ (top left), $t=2$ (top right), $t=3$ (bottom left), and $t=4$ (bottom right).
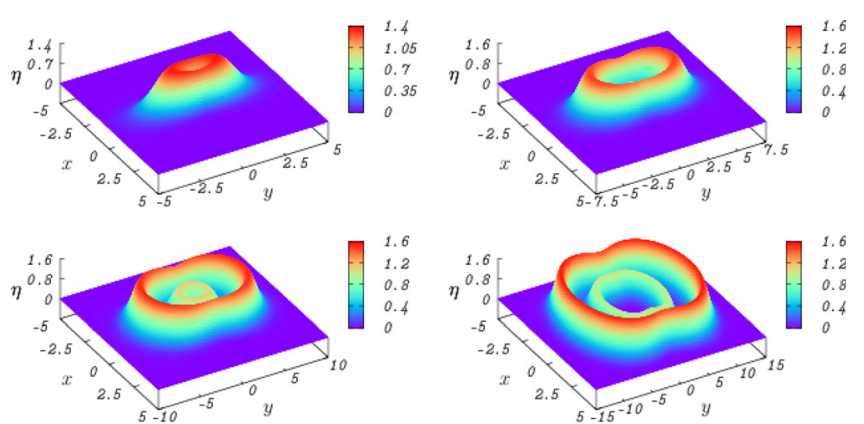

FIG. 13. Surface waves corresponding to the two-soliton solution of the ecKP-II equation with $k_{1}=0.5, k_{2}=-0.5, k_{3}=0.4$, and $k_{4}=-0.4$ for $t=0$ (top left), $t=0.5$ (top right), $t=1$ (bottom left), and $t=2$ (bottom right). 


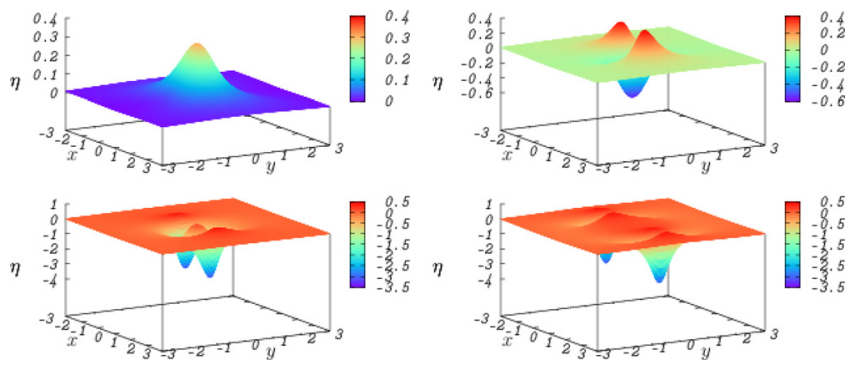

FIG. 14. Surface waves corresponding to the ecKP-I lump solution with $\kappa=0.25$, for $t=0$ (top left), $t=0.5$ (top right), $t=1$ (bottom left), and $t=2$ (bottom right).

It is easy to see that for $\sin \beta=0$, the wave elevation $\eta$ has the form

$$
\eta=-\frac{4}{6^{1 / 3}} \sqrt{\frac{a}{\gamma}} f(\zeta-3 \kappa \tau),
$$

where the function

$$
f(X)=\frac{4 \kappa\left(1-\kappa X^{2}\right)}{\left(1+\kappa X^{2}\right)^{2}}
$$

has one high maximum for $X=0$ and two weak minima for $X= \pm \sqrt{3 / \kappa}$, where

$$
f(0)=4 \kappa, \quad f( \pm \sqrt{3 / \kappa})=-\kappa / 2 .
$$

Therefore, for sufficiently large values of $t$, the wave elevation $\eta$ has two deep minima

$$
\eta_{\text {min }}=-\frac{16 \kappa}{6^{1 / 3}} \sqrt{\frac{a}{\gamma}}
$$

for

$$
\sin \beta=0, \quad \cosh \alpha=\frac{t}{\gamma\left(1+\frac{3 \kappa \Delta}{6^{1 / 3}}\right)} .
$$

The corresponding surface wave elevation $\eta$ is plotted in Fig. 14 for $\gamma=1, a=2, \Delta=1 / 2, W_{e}=2 / 3$, and $\kappa=0.25$.

\section{CONCLUDING REMARKS}

In this paper, we have derived and studied a new integrable version of the Kadomtsev-Petviashvili equation associated with the elliptic-cylindrical geometry of the wave fronts. The derivation was given in the context of surface gravity waves, but the equation can be readily derived in other physical contexts. We found transformations linking the derived model with the two classical versions of the KP equation, associated with the Cartesian and cylindrical geometries of the wave fronts, and the Lax pair for the new equation. We also completely classified approximate solutions for the surface gravity waves corresponding to the one-soliton solution of the ecKP equation, as well as discussing some other solutions.

In our derivation, a large distance variable has been used in preference to large time, although one can also use the large time variable. The dimensional form of the derived equation is given by

$$
\begin{aligned}
& {\left[2\left(\eta_{t}+\frac{c}{d} \eta_{\phi}\right)-\frac{3}{h_{0}} \eta \eta_{t}-\frac{h_{0}^{2}}{c^{2}}\left(\frac{1}{3}-W_{e}\right) \eta_{t t t}\right.} \\
& \left.+\frac{c}{d} \frac{\phi}{\phi^{2}-1} \eta+\frac{\psi^{2}}{\phi^{2}-1} \eta_{t}\right]_{t}-\frac{c^{2}}{d^{2}} \frac{1}{\phi^{2}-1} \eta_{\psi \psi}=0
\end{aligned}
$$

where $\eta$ is the free surface elevation, $t$ is time, $\phi=\cosh \alpha$ and $\psi=\sin \beta$ are variables related to the elliptic cylindrical coordinates, $h_{0}$ is the unperturbed fluid depth, $c=\sqrt{g h_{0}}$ is the linear long-wave speed, $d$ is half of the distance between the foci of the coordinate lines (say, the boundary of the wave source), and $W_{e}$ is the Weber number. The key nondimensional parameters used in the paper are expressed via the dimensional parameters as follows:

$$
A=\frac{d \lambda^{3} h_{s}^{6}}{h_{0}^{10}}, \quad \gamma=\frac{d}{\lambda}, \quad \Delta=\frac{\lambda^{2} h_{s}^{4}}{h_{0}^{6}},
$$

where $\lambda$ is the wave length, while $\delta=\frac{h_{0}}{\lambda}$ and $\epsilon=\frac{h_{s}}{h_{0}}$. To derive the ecKP equation, we required that $\Delta$ is a small parameter. We also note that for any given values of $A, \gamma$, and $\Delta$, there exists a range of the physical validity of the model, as can be seen from the expressions above.

The importance of the model to particular applications has not been discussed in this paper, and it is an open question at the moment. Another open question is the study of the wave instabilities within the framework of the ecKP equation, continuing the lines of research for the $\mathrm{KP}^{24}$ and cKP equations. ${ }^{25}$

In our paper, we considered only some simple solutions of the derived equation. Recently, there has been significant progress in the classification of soliton solutions of the KP equation with applications to water wave problems (see, Refs. 26-29 and references therein). It would be interesting to see the counterpart of this classification for the derived equation, and for the approximate solutions for surface waves.

The derivation of the ecKP equation from the full set of Euler equations opens the way to the study of internal and surface waves on a current for a stratified fluid, as well as accounting for the effects of variable background and Earth's rotation, which will be reported elsewhere. It paves the way for other applications, for example, in the context of matter waves in Bose-Einstein condensates (e.g., Refs. 18 and 19), since the hydrodynamic form of the Gross-Pitaevskii equation is similar to the problem formulation (1). Also, recent studies of "spherical nebulons,"30 based on the spherical KP equation, can be extended since the ellipsoidal KP equation, associated with the ellipsoidal coordinates, can be derived from the equations for "a dusty plasma" along the lines discussed in this paper.

Finally, it is natural to ask a question whether one can derive other versions of the KP equation, associated with other coordinate systems (i.e., with other wave geometries), and whether one can find the general description of all admissible maps of the type discussed in Sec. III, associated with the problem formulation (1).

\section{ACKNOWLEDGMENTS}

We thank G. A. El, E. V. Ferapontov, and R. H. J. Grimshaw for useful discussions, and referees for constructive 
comments and helpful references. K.R.K. and A.O.S. acknowledge support and hospitality of the Institut de Mathématiques de Bourgogne, where they held visiting positions in the springsummer of 2012, which has made this collaboration possible. C.K. and V.B.M. thank for financial support by the ANR via the program ANR-09-BLAN-0117-01.

\section{APPENDIX A: PROBLEM FORMULATION}

In the variables

$$
\begin{aligned}
\zeta & =\frac{\epsilon^{2}}{\delta^{2}}(\gamma \cosh \alpha-t), \\
R & =\frac{\epsilon^{6}}{\delta^{4}} \gamma \cosh \alpha, \quad \nu=\frac{\delta}{\epsilon^{2}} \sin \beta, \\
u & =\frac{\epsilon^{3}}{\delta^{2}} U, \quad v=\frac{\epsilon^{5}}{\delta^{3}} V, \quad w=\frac{\epsilon^{5}}{\delta^{4}} W, \\
\eta & =\frac{\epsilon^{3}}{\delta^{2}} H, \quad p=\frac{\epsilon^{3}}{\delta^{2}} P,
\end{aligned}
$$

the problem formulation (7)-(13) assumes the form

$$
\begin{aligned}
& -U_{\zeta}+P_{\zeta}+\Delta\left[U U_{\zeta}+W U_{z}+P_{R}\right. \\
& \left.-\frac{R-\sqrt{R^{2}-A^{2}}}{R^{2}-A^{2}}\left(\nu P_{\nu}+R \nu^{2} P_{\zeta}\right)\right]+O\left(\Delta^{2}\right)=0, \\
& -V_{\zeta}+\frac{1}{\sqrt{R^{2}-A^{2}}} P_{\nu}+\frac{R-\sqrt{R^{2}-A^{2}}}{\sqrt{R^{2}-A^{2}}} \nu P_{\zeta} \\
& +\Delta\left[U V_{\zeta}+W V_{z}+\frac{R-\sqrt{R^{2}-A^{2}}}{\sqrt{R^{2}-A^{2}}} \nu P_{R}\right. \\
& +\frac{\left(R-\sqrt{R^{2}-A^{2}}\right)\left(R^{2}+A^{2}\right)}{2\left(R^{2}-A^{2}\right)^{3 / 2}} \nu^{3} P_{\zeta} \\
& \left.-\frac{\left(R-\sqrt{R^{2}-A^{2}}\right)^{2}+R^{2}}{2\left(R^{2}-A^{2}\right)^{3 / 2}} \nu^{2} P_{\nu}\right]+O\left(\Delta^{2}\right)=0, \\
& P_{z}-\Delta W_{\zeta}+O\left(\Delta^{2}\right)=0, \\
& U_{\zeta}+W_{z}+\Delta\left[U_{R}-\frac{R-\sqrt{R^{2}-A^{2}}}{R^{2}-A^{2}}\left(R \nu^{2} U_{\zeta}+\nu U_{\nu}\right)\right. \\
& \left.+\frac{1}{\sqrt{R^{2}-A^{2}}}\left(\left(R-\sqrt{R^{2}-A^{2}}\right) \nu V_{\zeta}+V_{\nu}+U\right)\right] \\
& +O\left(\Delta^{2}\right)=0, \\
& \left.P\right|_{z=1+\Delta H(\zeta, R, \mu)}=H-\Delta W_{e} H_{\zeta \zeta}+O\left(\Delta^{2}\right), \\
& \left.W\right|_{z=1+\Delta H(\zeta, R, \mu)}=-H_{\zeta}+\Delta U H_{\zeta}+O\left(\Delta^{2}\right), \\
& \left.W\right|_{z=0}=0,
\end{aligned}
$$

where $\Delta=\frac{\epsilon^{4}}{\delta^{2}}$ and $A=\gamma \frac{\epsilon^{6}}{\delta^{4}}$. Here, we have not shown the explicit form of the higher-order terms in the small parameter $\Delta$ (denoted by $O\left(\Delta^{2}\right)$ ) since these terms are not needed in the derivation of our asymptotic equation.

\section{APPENDIX B: QUASI-PERIODIC SOLUTIONS}

Quasi-periodic (multiphase) solutions to the KP equation can be given in terms of multi-dimensional theta functions on compact Riemann surfaces of arbitrary genus $n$ (see, Ref. 31) in the form

$$
U(\xi, Y, \tau)=2 \partial_{x}^{2} \ln \Theta[\xi p+Y v+\tau q+l]+C,
$$

where $\Theta$ is the Riemann theta function, $p, v, q$, and $l$ are periods of certain integrals on this surface, and $C$ is constant with respect to the coordinates $\xi, Y$, and $\tau$ (see Refs. 22 and 33 for details). For a given Riemann surface and a given point on it, these quantities are uniquely determined.

In genus 2, all such surfaces are hyperelliptic. In this case, we consider the hyperelliptic curve with branch points $-1,-2,-3,0,1$, and 2 . These solutions are numerically evaluated with the spectral code by Frauendiener and
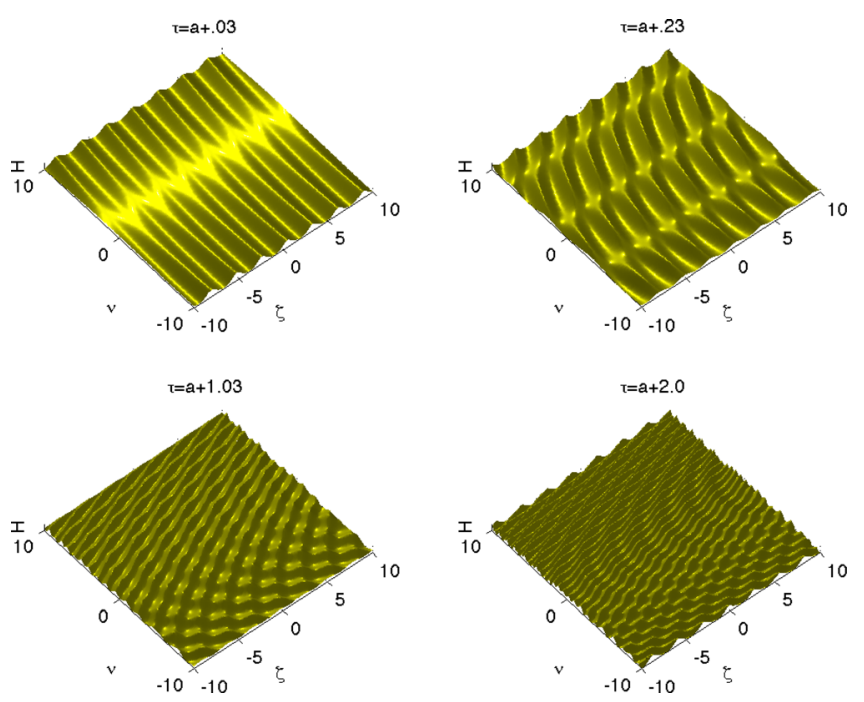

FIG. 15. Genus 2 solution (42) to the ecKP-II equation for $a=0.01$ generated by the curve $w^{2}=\prod_{i=1}^{6}\left(z-e_{i}\right), e_{1}=-3, e_{2}=-2, e_{3}=-1, e_{4}=$ $0, e_{5}=1, e_{6}=2$ for several values of $\tau$.
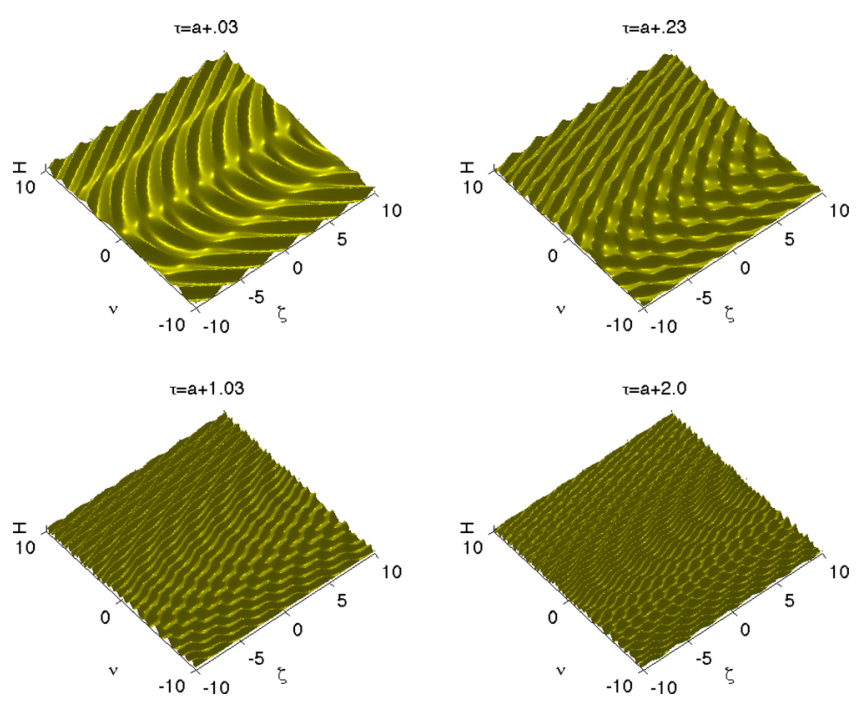

FIG. 16. Genus 2 solution (42) to the ecKP-II equation for $a=1$ generated by the curve $w^{2}=\prod_{i=1}^{6}\left(z-e_{i}\right), e_{1}=-3, e_{2}=-2, e_{3}=-1, e_{4}=0, e_{5}=$ $1, e_{6}=2$ for several values of $\tau$. 

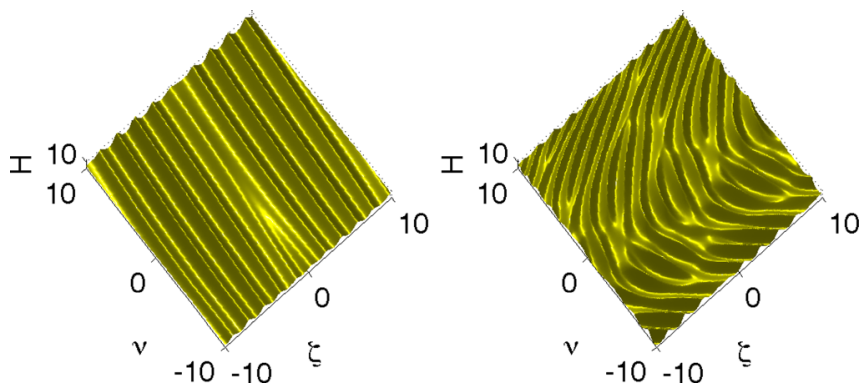

FIG. 17. Genus 3 solution (42) to the ecKP-II equation for $a=0.01$ (left) and $a=1$ (right), generated by the curve $w^{2}=\prod_{i=1}^{8}\left(z-e_{i}\right), e_{1}$ $=-5, e_{2}=-4, e_{3}=-3, e_{4}=-2, e_{5}=-1, e_{6}=0, e_{7}=1, e_{8}=2$ at $\tau=a$ +0.01 .

Klein. ${ }^{32,33}$ The related solutions to the ecKP-II equation are generated from the corresponding solutions of the KP-II equation via the map (30) with $a=0.01$. We clearly see in Fig. 15 the formation of intersecting families of parabolic fronts.

In the same setting with $a=1$, i.e., a theta-functional solution to the ecKP-II equation, the formation of curved profiles is already present for small $\tau-a$ as can be seen in Fig. 16. Both cases asymptotically coincide for $\tau \rightarrow \infty$.

In higher genus, the solutions are $g$-phase solutions, i.e., they have more structure as can be seen in Fig. 17. We consider here again hyperelliptic surfaces. The close to cKP solutions are for small time essentially independent of the transversal coordinate.

${ }^{1}$ B. P. Kadomtsev and V. I. Petviashvili, Sov. Phys. Dokl. 15, 539 (1970).

${ }^{2}$ M. J. Ablowitz and H. Segur, J. Fluid Mech. 92, 691 (1979).

${ }^{3}$ R. S. Johnson, J. Fluid Mech. 97, 701 (1980).

${ }^{4}$ M. J. Ablowitz and H. Segur, Solitons and the Inverse Scattering Transform (SIAM, Philadelphia, 1981).

${ }^{5} \mathrm{R}$. S. Johnson, A Modern Introduction to the Mathematical Theory of Water Waves (Cambridge University Press, Cambridge, 1997).

${ }^{6}$ M. J. Ablowitz, Nonlinear Dispersive Waves: Asymptotic Analysis and Solitons (Cambridge University Press, Cambridge, 2011).
${ }^{7}$ V. D. Lipovskii, V. B. Matveev, and A. O. Smirnov, J. Sov. Math. 46, 1609 (1989).

${ }^{8}$ V. I. Golin'ko, V. S. Dryuma, and Yu. A. Stepanyants, "Nonlinear quasicylindrical waves: Exact solutions of the cylindrical KadomtsevPetviashvili equation," in Proc. 2nd Int. Workshop on Nonlinear and Turbulent Processes in Physics, Kiev, 1983 (Harwood Acad., Gordon and Breach, 1984). pp. 1353-1360.

${ }^{9}$ C. Klein, V. B. Matveev, and A. O. Smirnov, Theor. Math. Phys. 152, 1132 (2007).

${ }^{10}$ Yu. A. Stepanyants, Russ. Math. Surv. 44, 255 (1989).

${ }^{11}$ A. A. Lugovtsov and B. A. Lugovtsov, Dyn. Contin. Medium (Novosibirsk) 1, 195 (1969) (in Russian).

${ }^{12}$ V. S. Dryuma, Dokl. Akad. Nauk SSSR 268, 15 (1983).

${ }^{13}$ R. H. J. Grimshaw, L. A. Ostrovsky, V. I. Shrira, and Yu. A. Stepanyants, Surv. Geophys. 19, 289 (1998).

${ }^{14}$ R. H. J. Grimshaw, "Internal solitary waves," in Environmental Stratified Flows, edited by R. Grimshaw (Kluwer, Boston, 2001), p. 1-28.

${ }^{15}$ S. P. Novikov, S. V. Manakov, L. P. Pitaevskii, and V. E. Zakharov, Theory of Solitons: The Inverse Scattering Method (Springer-Verlag, 1984).

${ }^{16}$ R. Grimshaw, Stud. Appl. Math. 73, 1 (1985).

${ }^{17}$ V. D. Lipovskii, Izv. Akad. Nauk SSSR, Fiz. Atmos. Okeana 21, 864 (1985).

${ }^{18} \mathrm{G}$. Huang, "Nonlinear amplitude equations and soliton excitations in Bose-Einstein condensates," in Nonlinear Waves in Fluids: Recent Advances and Modern Applications, CISM Courses and Lectures Vol. 483, edited by R. Grimshaw (Springer, 2004), pp. 169-196.

${ }^{19}$ S. Tsuchiya, F. Dalfovo, and L. Pitaevsky, Phys. Rev. A 77, 045601 (2008).

${ }^{20}$ Y.-T. Gao and B. Tian, Phys. Lett. A 349, 314 (2006).

${ }^{21}$ V. B. Matveev and M. A. Salle, Darboux Transformations and Solitons (Springer, Berlin, 1991).

${ }^{22}$ E. D. Belokolos, A. I. Bobenko, V. Z. Enol'skii, A. R. Its, and V. B. Matveev, Algebro-Geometric Approach to Nonlinear Integrable Equations (Springer, Berlin, 1994).

${ }^{23}$ J. W. Miles, J. Fluid Mech. 79, 171 (1977).

${ }^{24}$ V. E. Zakharov, JETP Lett. 22, 172 (1975).

${ }^{25}$ L. A. Ostrovsky and V. I. Shrira, Sov. Phys. JETP 44, 738 (1976).

${ }^{26}$ G. Biondini and S. Chakravarty, J. Math. Phys. 47, 033514 (2006).

${ }^{27}$ G. Biondini, Phys. Rev. Lett. 99, 064103 (2007).

${ }^{28}$ S. Chakravarty and Y. Kodama, Stud. Appl. Math. 123, 83 (2009).

${ }^{29}$ H. Yeh, W. Li, and Y. Kodama, Eur. Phys. J. Spec. Top. 185, 97 (2010).

${ }^{30}$ Y.-T. Gao and B. Tian, EPL 77, 15001 (2007).

${ }^{31}$ I. M. Krichever, Russ. Math. Surv. 32, 185 (1977).

${ }^{32}$ J. Frauendiener and C. Klein, J. Comput. Appl. Math. 167, 193 (2004).

${ }^{33}$ J. Frauendiener and C. Klein, Lett. Math. Phys. 76, 249 (2006). 